\title{
Double-bromo and extraterminal (BET) domain proteins regulate dendrite morphology and mechanosensory function
}

\author{
Joshua A. Bagley, ${ }^{1,2,3,4}$ Zhiqiang Yan,,3,4 Wei Zhang, ${ }^{2,3,4}$ Jill Wildonger, ${ }^{2,3,4,5}$ Lily Yeh Jan, ${ }^{2,3,4}$ \\ and Yuh Nung Jan ${ }^{2,3,4}$ \\ ${ }^{1}$ Neuroscience Graduate Program, University of California at San Francisco, San Francisco, California 94158, USA; ${ }^{2}$ Howard \\ Hughes Medical Institute, ${ }^{3}$ Department of Physiology, ${ }^{4}$ Department of Biochemistry and Biophysics, University of California at \\ San Francisco at San Francisco, California 94158, USA
}

\begin{abstract}
A complex array of genetic factors regulates neuronal dendrite morphology. Epigenetic regulation of gene expression represents a plausible mechanism to control pathways responsible for specific dendritic arbor shapes. By studying the Drosophila dendritic arborization (da) neurons, we discovered a role of the double-bromodomain and extraterminal (BET) family proteins in regulating dendrite arbor complexity. A loss-of-function mutation in the single Drosophila BET protein encoded by female sterile 1 homeotic [fs(1)h] causes loss of fine, terminal dendritic branches. Moreover, $f_{s}(\mathbf{1}) \mathrm{h}$ is necessary for the induction of branching caused by a previously identified transcription factor, $\mathrm{Cut}(\mathrm{Ct})$, which regulates subtype-specific dendrite morphology. Finally, disrupting $f_{s}(1) h$ function impairs the mechanosensory response of class III da sensory neurons without compromising the expression of the ion channel NompC, which mediates the mechanosensitive response. Thus, our results identify a novel role for BET family proteins in regulating dendrite morphology and a possible separation of developmental pathways specifying neural cell morphology and ion channel expression. Since the BET proteins are known to bind acetylated histone tails, these results also suggest a role of epigenetic histone modifications and the "histone code," in regulating dendrite morphology.
\end{abstract}

[Keywords: female sterile 1 homeotic; double bromodomain and extraterminal; histone acetylation; epigenetics; dendrite morphogenesis; mechanosensory function]

Supplemental material is available for this article.

Received February 13, 2014; revised version accepted August 4, 2014.

Dendrites are the primary site of information input to neural circuits, and the shape of dendritic arbors influences the electrophysiological responses of neurons (Häusser and Mel 2003). Due to the existence of highly diverse morphologies among different neuronal subtypes, a question of the relationship between form and function arises: By understanding how the shape of a neuron is specified, we can understand how morphology relates to neural function and how altered morphology relates to dysfunction.

Neurons can be defined by their physiology, morphology, and gene expression. Neuronal diversity is thought to arise from the combinatorial expression of genetic determinants (Hobert et al. 2010). The dendritic arborization (da) sensory neurons of the Drosophila peripheral nervous system (PNS) constitute a powerful system to

\footnotetext{
${ }^{5}$ Present address: Biochemistry Department, University of WisconsinMadison, Madison, Wisconsin 53706, USA

Corresponding author: yuhnung.jan@ucsf.edu

Article is online at http://www.genesdev.org/cgi/doi/10.1101/gad.239962.114.
}

study genetic determinants of dendritic arbor morphology (Corty et al. 2009; Jan and Jan 2010). In particular, the use of Drosophila genetic techniques to study the specification of stereotyped, subtype-specific dendritic arbor shapes (Grueber et al. 2002) resulted in the identification of multiple transcription factors, encoded by abrupt $(a b)$, knot/collier $(\mathrm{kn} / \mathrm{col})$, spineless $(\mathrm{ss})$, and cut $(\mathrm{ct})$, which regulate dendritic arbor morphology (Grueber et al. 2003; Li et al. 2004; Sugimura et al. 2004; Kim et al. 2006; Hattori et al. 2007; Crozatier and Vincent 2008). However, large-scale genomic analyses comparing the transcriptomes of various neural subtypes indicate a daunting amount of varied gene expression and implicate regulation by multiple transcription factors (Sugino et al. 2006; Hattori et al. 2013; Iyer et al. 2013). Thus, a particular

(c) 2014 Bagley et al. This article is distributed exclusively by Cold Spring Harbor Laboratory Press for the first six months after the full-issue publication date (see http://genesdev.cshlp.org/site/misc/terms.xhtml). After six months, it is available under a Creative Commons License (Attribution-NonCommercial 4.0 International), as described at http:// creativecommons.org/licenses/by-nc/4.0/. 
neuronal morphology is likely the result of coordination between multiple genomic programs.

Epigenetic modifications are one mechanism that could allow coordinated, genome-wide expression profiles. Chromatin is packaged into nucleosomes, where DNA nucleotides wrap an octamer of histone proteins. The chromatin structure can be altered through three main types of modifications, consisting of direct methylation of DNA nucleotides, post-translational histone-tail modifications such as acetylation and methylation, and ATP-dependent chromatin remodeling (Ho and Crabtree 2010). ATP-dependent chromatin remodelers were first shown to regulate dendrite morphology when RNAi knockdown of brahma (brm)-associated protein $60 \mathrm{kD}$ (Bap60), Bap55, and the ATPase brm altered the dendritic arbors of class I da sensory neurons (Parrish et al. 2006). In mammalian neurons, the neural-specific Brg/Brm-associated factor (BAF) complex (nBAF), which contains BAF53b and the ATPase Brg, regulates activity-dependent dendrite growth (Wu et al. 2007). In addition, the Drosophila BAF53a/b homolog Bap55 regulates dendritic targeting of olfactory projection neurons (PNs) (Tea and Luo 2011).

The post-translational modification of histone tails involves three types of molecules: The "writers" add methyl, acetyl, or phospho groups and consist of histone methyl transferase (HMT), histone acetyltransferase (HAT), and kinase enzymes. The "erasers" remove these modifications and include demethylases (DMTs), histone deacetyltransferases (HDACs), and phosphatases. Finally, the "readers" are scaffolding proteins that recognize and bind acetyl, methyl, or phosphate modifications to position the "writer" and "eraser" enzymes along with transcriptional machinery to the correct genomic position and thereby modify gene expression (Borrelli et al. 2008). The discovery that the Polycomb repressor complex, which binds methylated histone tails, regulates da sensory neuron dendrite morphology indicates a role of histone methylation in dendrite development (Parrish et al. 2007) and a notion supported by the recent finding that the chromodomain Y-like (CDYL) protein negatively regulates dendritic complexity (Qi et al. 2014). Regarding a role of histone acetylation in dendrite morphogenesis, both HDAC and HAT activities have been implicated in regulating dendrite morphology. Specifically, the Drosophila $\mathrm{HDACl} / 2$ homolog Rpd3 regulates class I da sensory neuron morphology (Parrish et al. 2006) and olfactory PN dendritic targeting (Tea et al. 2010). In addition, HDAC2 suppresses dendritic spine density of hippocampal CA1 and dentate granule neurons (Guan et al. 2009). The HAT enzyme Pcaf also regulates class I da sensory neuron dendrite morphology (Parrish et al. 2006). A different HAT enzyme, CREB-binding protein $(\mathrm{CBP})$, regulates the developmental pruning of class IV da sensory neuron dendrites (Kirilly et al. 2011), and mutations in the human homolog CREBBP cause the mental retardation syndrome Rubenstein-Taybi (Petrij et al. 1995). While these studies indicate a definite role of "writers" and "erasers" of histone modifications in regulating dendrite morphogenesis, the role of "reader" scaf- folding proteins associated with histone acetylation has not been thoroughly investigated.

Double-bromo and extraterminal (BET) domain-containing proteins bind acetylated histone tails (Umehara et al. 2010a,b) and modulate gene expression (Kanno et al. 2004; Sinha et al. 2005; Denis et al. 2006; Chang et al. 2007). In mice, mutations in one BET family member, BRD2, cause neural tube closure defects, behavioral abnormalities, and altered interneuron numbers (Gyuris et al. 2009; Shang et al. 2009; Velíšek et al. 2011). In addition, in certain human genomic population studies, mutations in BRD2 have been associated with juvenile myoclonic epilepsy (Pal et al. 2003) and photosensitivity, which is frequently observed in idiopathic generalized epilepsies (Lorenz et al. 2006). In the current study, we provide evidence for a role of the Drosophila homolog of BRD2, encoded by female sterile 1 homeotic $\left[f_{s}(1) h\right]$, in regulating dendrite morphology and sensory function.

\section{Results}

$\mathrm{fs}(1) \mathrm{h}^{1112}$ reduces higher-order dendritic arbor complexity

The $f_{S}(1) h^{1112}$ allele was created by ethyl methanesulfonate (EMS) mutagenesis during a previous forward genetic screen of lethal X-chromosome mutations (Zheng et al. 2008). To circumvent the early embryonic lethality associated with this allele and assess the cell-autonomous function of $f_{S}(1) h$ in dendritic development, we employed mosaic analysis with a repressible cell marker (MARCM) (Lee and Luo 1999). This technique allows the generation of homozygous mutant neuron clones marked by GAL4-driven fluorescent proteins within an essentially heterozygous animal. Using the pan-da neuron GAL4 ${ }^{109(2) 80}$ driver (Gao et al. 1999) to express membrane-bound UAS-mCD8-GFP, we visualized the dendritic arbors of single-cell sensory neuron clones within live, intact third instar Drosophila larvae. We analyzed these dendritic arbors through confocal imaging followed by digital reconstruction and compared the morphologies of the $f_{S}(1) h$ mutant and wild-type control clones.

The da sensory neurons consist of four subtypes (classes) of neurons (class I, II, III, and IV), categorized by their dendritic arbor morphology (Grueber et al. 2002). The $f_{S}(1) h^{1112}$ mutation alters the normal morphology of different classes of da neurons to a varying degree. In class I and class II da neurons, the dendritic arbor phenotype was mild but significant, causing a decrease $(\sim 13 \%)$ in total class I da neuron dendritic length [control: $1947 \mu \mathrm{m} \pm 57 \mu \mathrm{m}, n=5$ cells vs. $f_{S}(1) h^{1112}$ : $1699 \mu \mathrm{m} \pm 66 \mu \mathrm{m}, n=4$ cells; $P=0.028$ ] (Supplemental Fig. S1G) and an $\sim 41 \%$ decrease in class II da neuron branch points [control: $40 \pm 5$ branch points, $n=6$ cells vs. $f_{S}(1) h^{1112}: 23 \pm 3$ branch points, $n=5$ cells; $\left.P=0.017\right]$ (Supplemental Fig. S1H). There was no significant change in class I da neuron branch points [control: $30 \pm 1$ branch points, $n=5$ cells vs. $f_{S}(1) h^{1112}: 29 \pm 3$ branch points, $n=$ 4 cells; $P=0.937$ ] (Supplemental Fig. S1G) or class II da neuron dendritic length [control: $2009 \mu \mathrm{m} \pm 84 \mu \mathrm{m}, n=6$ 
cells vs. $f_{S}(1) h^{1112}: 1818 \mu \mathrm{m} \pm 214 \mu \mathrm{m}, n=5$ cells; $P=$ 0.560] (Supplemental Fig. S1H). In contrast, we observed a stronger phenotype in the more complex arbors of class IV da neurons (Supplemental Fig. S2). The class IV da neuron dendritic arbors were severely reduced by $\sim 64 \%$ in total length $\left[f_{S}(1) h^{1112}: 5514 \mu \mathrm{m} \pm 804 \mu \mathrm{m}, n=3\right.$ cells vs. control: $15,430 \mu \mathrm{m} \pm 428 \mu \mathrm{m}, n=3$ cells; $P<0.001]$ (Supplemental Fig. S2E) and had $\sim 74 \%$ fewer branch points $\left[f_{S}(1) h^{1112}: 129 \pm 26\right.$ branch points, $n=3$ cells vs. control: $488 \pm 17$ branch points, $n=3$ cells; $P<0.001]$ (Supplemental Fig. S2F). As indicated by a Sholl analysis (Supplemental Fig. S2G), this reduction in branching occurred uniformly throughout the dendritic arbor.

The most striking phenotype was observed in class III da neuron dendritic arbors, which are characterized by small, filopodia-like protrusions ("spikes") sprouting from their primary dendrites (Grueber et al. 2002) and giving them a furry appearance. Whereas the overall arbor size of wild-type (Fig. 1A) and $f_{S}(1) h^{1112}$ class III da neurons (Fig. 1B) appeared similar, the dendritic spikes were nearly completely abolished in $f_{S}(1) h^{1112}$ neurons (Fig. $\left.1 \mathrm{~A}^{\prime}, \mathrm{B}^{\prime}\right)$. Indeed, $f_{S}(1) h^{1112}$ caused a severe $(>90 \%)$ reduction of the spikes compared with control $\left[f_{S}(1) h^{1112}\right.$ : $24 \pm 2$ spikes, $n=8$ cells vs. control: $261 \pm 45$ spikes, $n=6$ cells; $P<0.001$ ] (Fig. 1E). Dendritic arbors consist of lower-order, thicker, primary dendrites and higher-order, thinner, fine dendritic terminal branches. The class III spikes represent the higher-order, fine dendritic branches, but we also assessed whether $f_{S}(1) h^{1112}$ altered primary branch morphology. We observed a smaller (26\%) but significant reduction in primary dendrite branch length $\left[f_{s}(1) h^{1112}: 2107 \mu \mathrm{m} \pm 137 \mu \mathrm{m}, n=8\right.$ cells vs. control: $2830 \mu \mathrm{m} \pm 184 \mu \mathrm{m}, n=6$ cells; $P=0.011$ ] (Fig. 1F). We also observed a significant decrease $(39 \%)$ in primary dendrite branch point number $\left[f_{s}(1) h^{1112}: 11 \pm 1\right.$ branch points, $n=8$ cells vs. control: $18 \pm 2$ branch points, $n=6$ cells; $P=0.008$ ] (Fig. 1G), but this reduction was much less severe than the nearly complete reduction in spike morphology. Therefore, considering the phenotypes observed in all classes of da neurons, the $f_{S}(1) h^{1112}$ mutation appears to affect mainly the smaller, terminal dendritic protrusions, and this effect is stronger in dendritic arbors of higher complexity. Since the class III da neurons are responsive to gentle touch stimuli and their touch sensitivity can be measured through in vitro recordings (Yan et al. 2013), we decided to focus on the role of $f_{S}(1) h$ in regulating both the structure and the function of class III dendritic arbors.

There are two splice isoforms of $f_{S}(1) h$ : a short (Fsh-S) and a long (Fsh-L) isoform (Supplemental Fig. S3A). Defects in embryonic segmental transformations caused by $f_{S}(1) h$ mutations can be rescued by expressing Fsh-S (Chang et al. 2007). Therefore, we attempted to rescue the class III dendritic arbor phenotype of $f_{S}(1) h^{1112}$ with a UAS transgene containing Fsh-S. Expressing UAS-Fsh-S resulted in a partial rescue $(57 \%$ of control) of the reduction of spikes $\left[f_{S}(1) h^{1112} ; U A S-F s h-S: 149 \pm 15\right.$ spikes, $n=8$ cells vs. $f_{S}(1) h^{1112}: 24 \pm 2$ spikes, $n=8$ cells; $P=0.004$ ] (Fig. 1E) caused by $f_{S}(1) h^{1112}$ with a negligible effect on primary branch length $\left[f_{S}(1) h^{1112} ; U A S-F s h-S\right.$ :
$2316 \mu \mathrm{m} \pm 81 \mu \mathrm{m}, n=8$ cells vs. $f_{S}(1) h^{1112}: 2107 \mu \mathrm{m} \pm$ $137 \mu \mathrm{m}, n=8$ cells; $P=0.712$ ] (Fig. $1 \mathrm{~F}$ ), or primary dendrite branch point number $\left[f_{S}(1) h^{1112} ; U A S-F s h-S\right.$ : $13 \pm 1$ branch points, $n=8$ cells vs. $f_{S}(1) h^{1112}: 11 \pm 1$ branch points, $n=8$ cells; $P=0.662$ ] (Fig. 1G). To look for possible effects of Fsh-S overexpression using the GAL4/ UAS system, we examined the arbors of class III da neurons with UAS-Fsh-S expression in wild-type and $f_{S}(1) h$ backgrounds (Fig. 1D). The expression of UAS-Fsh-S in a wild-type background caused a reduction of spike number [UAS-Fsh-S: $136 \pm 27$ spikes, $n=7$ cells vs. control: $261 \pm 45$ spikes, $n=6$ cells; $P=0.01$ ] (Fig. $1 \mathrm{E}$ ) but no change in primary branch length [UAS-Fsh-S: $2709 \mu \mathrm{m} \pm 183 \mu \mathrm{m}$, $n=7$ cells vs. control: $2830 \mu \mathrm{m} \pm 184 \mu \mathrm{m}, n=6$ cells; $P=0.945$ ] (Fig. 1F) or branch point number [UAS-Fsh-S: $15 \pm 2$ branch points, $n=7$ cells vs. control: $18 \pm 2$ branch points, $n=6$ cells; $P=0.442$ ] (Fig. 1G). These findings indicate that the dendritic arbor defect in spike morphology caused by $f_{S}(1) h^{1112}$ can be partially rescued by expression of $U A S-F s h$-S.

The rescue of the $f_{S}(1) h^{1112}$ dendrite phenotype by expression of $U A S-F s h-S$ is only a partial rescue. Moreover, UAS-Fsh-S expression in wild-type da neurons caused a reduction of dendritic spikes. One possible explanation is that Fsh-S is required at a specific expression level such that either too little or too much expression would alter dendritic morphogenesis, as is observed with the transcription factor Ct (Grueber et al. 2003). Since the rescue employs GAL4/UAS-mediated expression of Fsh-S, the expression level is likely higher than endogenous wild-type expression levels. To address this possibility, we took advantage of the temperature dependence of GAL4/UAS activity (Duffy 2002). In our initial experiments, all flies were raised at $25^{\circ} \mathrm{C}$, but we posited that moderating the expression level may yield a more favorable rescue. Therefore, we repeated the same experiment while raising the flies at a lower temperature $\left(20^{\circ} \mathrm{C}\right)$ to attenuate GAL4/UAS expression (Duffy 2002). We observed a significant increase in the number of class III da neuron spikes in the dendritic arbors of neurons expressing UAS-Fsh-S from both $f_{S}(1) h^{1112}\left[f_{S}(1) h^{1112}\right.$; UAS-Fsh-S/+, $25^{\circ} \mathrm{C}: 149 \pm 15$ spikes, $n=8$ cells vs. $20^{\circ} \mathrm{C}$ : $202 \pm 10$ spikes, $n=9$ cells; $P=0.004$ ] (Supplemental Fig. S4) and wild-type [UAS-Fsh-S/+, $25^{\circ} \mathrm{C}: 136 \pm 27$ spikes, $n=7$ cells vs. $20^{\circ} \mathrm{C}$ : $198 \pm 7$ spikes, $n=9$ cells; $P=$ 0.032] (Supplemental Fig. S4) neurons. However, we still observed only a partial rescue of the $f_{S}(1) h^{1112}$ reduced spike phenotype with UAS-Fsh-S expression at $20^{\circ} \mathrm{C}$ $\left[f_{S}(1) h^{1112} ; U A S-F s h-S /+, 20^{\circ} \mathrm{C}: 202 \pm 10\right.$ spikes, $n=9$ cells vs. control, $20^{\circ} \mathrm{C}$ : $319 \pm 20$ spikes, $n=8$ cells; $\left.P<0.001\right]$ (Supplemental Fig. S4) and a reduced spike phenotype with UAS-Fsh-S expression in a wild-type background at $20^{\circ} \mathrm{C}$ [UAS-Fsh-S/+, 20 ${ }^{\circ} \mathrm{C}: 198 \pm 7$ spikes, $n=9$ cells vs. control, $20^{\circ} \mathrm{C}: 319 \pm 20$ spikes, $n=8$ cells; $\left.P<0.001\right]$ (Supplemental Fig. S4). This suggests that Fsh-S is probably still overexpressed at $20^{\circ} \mathrm{C}$. We were unable to use a lower temperature for these experiments because, at $20^{\circ} \mathrm{C}$, we observed a variable reduction of GFP intensity between animals, which caused an inability to visualize the morphology of some neurons for quantification. Our observa- 


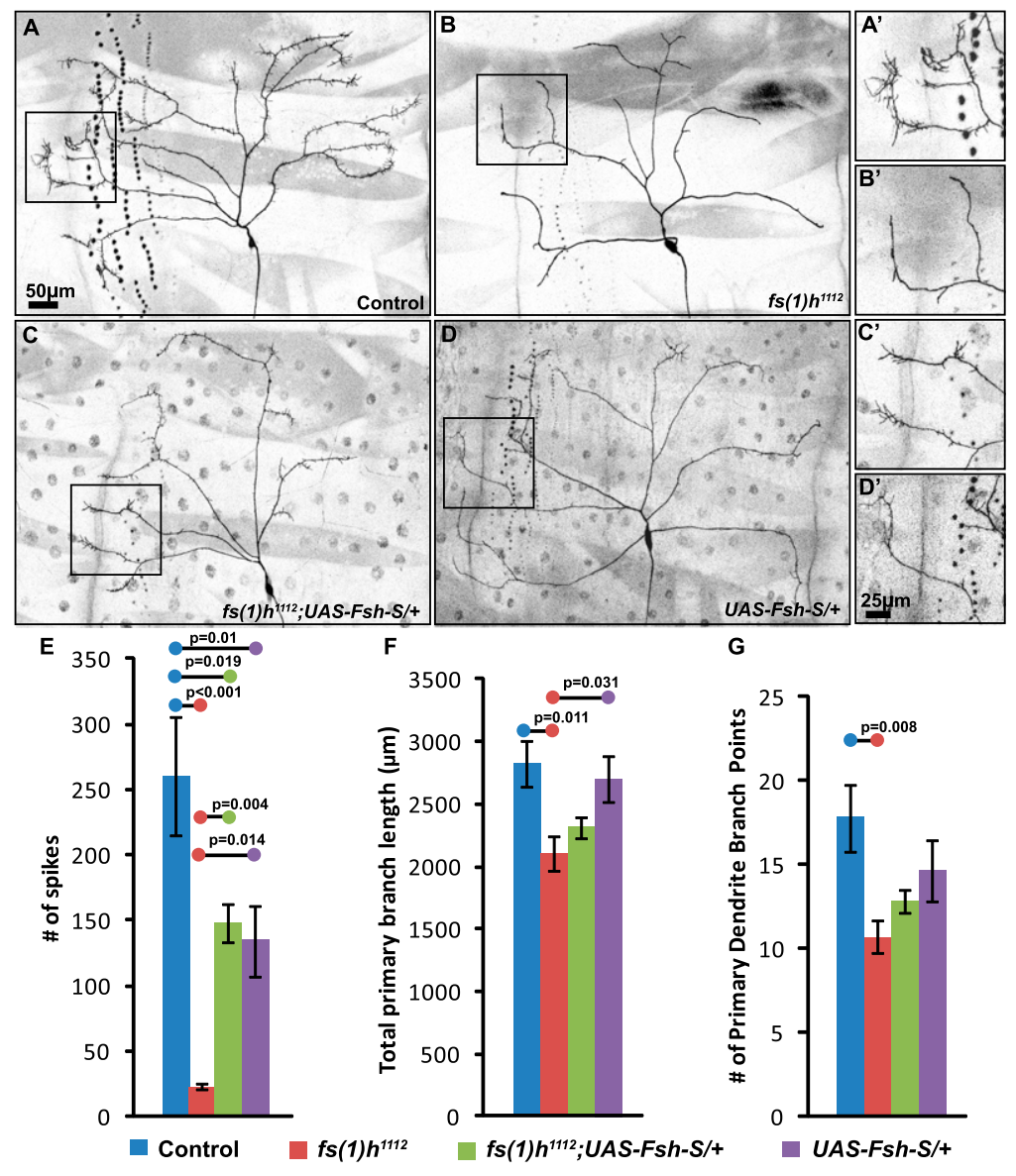

Figure 1. $f_{S}(1) h$ mutant neurons display reduced dendritic arbor branching. $(A-D)$ Dendritic arbors of class III da neuron (ddaF) MARCM clones in control

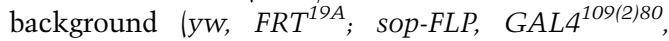
$U A S-m C D 8-G F P /+)$ and crossed to various mutant and transgenic flies as indicated. Bar, $50 \mu \mathrm{m} .\left(A^{\prime}-D^{\prime}\right)$ Magnified view representing the boxed regions in $A-$ $D$ showing a loss of terminal branching (spikes) in $f_{S}(1) h^{1112}$ mutants $\left(B^{\prime}\right)$ compared with the control $\left(A^{\prime}\right)$. The spikes return when UAS-Fsh-S is introduced $\left(C^{\prime}\right)$ and is similar to UAS-Fsh-S overexpression $\left(D^{\prime}\right)$. Bar, $25 \mu \mathrm{m} .(E-G)$ Quantification of dendritic arbor morphology. $(E) f_{S}(1) h^{1112}$ causes a nearly complete loss of spikes, which is rescued by UAS-Fsh-S. fs $(1) h^{1112}$ neurons also show a modest decrease in primary dendrite branch length $(F)$ and primary branch points $(G)$, which is not rescued by UAS-Fsh-S expression. The values are reported as mean \pm SEM, and $P$-value statistical significance is reported by results of one-way ANOVA followed by post-hoc Tukey test. tions of GAL4/UAS expression at two different temperatures indicate that dendrite morphology is sensitive to the level of Fsh-S expression and that both too little and too much expression causes abnormal dendrite morphology.

\section{$\mathrm{fs}(1) \mathrm{h}^{1112}$ causes loss of Fsh-S expression in da sensory neurons}

Since there are two protein isoforms of $f_{S}(1) h$ and we observed a partial rescue of $f_{S}(1) h^{1112}$ dendrite arbor complexity with expression of UAS-FSh-S, we wanted to determine the localization of endogenous Fsh protein. We addressed this question through the use of antibody staining against $f_{S}(1) h$ protein products (anti-Fsh). Two antibodies (Supplemental Fig. S3A) were used that recognize either both the short and long isoforms (anti-Fsh-S+L) or only the long isoform (anti-Fsh-L) (Chang et al. 2007). Consistent with a previous study (Chang et al. 2007), nuclear staining intensity was observed with antiFsh-S+L (Fig. 2A-C). However, we failed to detect any specific signal with anti-Fsh-L (Supplemental Fig. S5A-C). Anti-Fsh-S+L staining was observed in all da sensory neurons as well as epithelial cell nuclei (Fig. 2A-C). Thus, Fsh protein is expressed in all da sensory neurons.

To identify the Fsh isoforms expressed by the da neurons, we repeated the immunohistochemical experiments using a mutant $\left[f_{S}(1) h^{17}\right]$ that lacks Fsh-L but retains Fsh-S (Chang et al. 2007). In $f_{S}(1) h^{17}$ hemizygous mutants, the diffuse labeling of anti-Fsh-L (Supplemental Fig. S5G-I) appeared identical to the pattern observed in $f_{S}$ (1) $h^{17} /+$ heterozygous (Supplemental Fig. S5D-F) and wildtype (Supplemental Fig. S5A-C) larvae. Therefore, we conclude that the anti-Fsh-L labeling is nonspecific in the larval PNS tissue. Moreover, anti-Fsh-S+L labeling exhibited nearly identical patterns of staining in $f_{S}(1) h^{17}$ hemizygous mutants (Fig. 2G-I) and wild-type (Fig. 2A-C) or $f_{S}(1) h^{17} /+$ heterozygous (Fig. 2D-F) larvae. Therefore, we conclude that the da neurons express Fsh-S but probably not Fsh-L.

While these data strongly suggest that the da neurons do not express Fsh-L, we wanted to test the functional contribution of Fsh-L to class III da neuron morphology. Using GAL4 $4^{19-12}$ to express UAS-CD4-tdGFP in class III da sensory neurons, we visualized their dendritic arbor morphology in $f_{S}(1) h^{17}$ mutants. Overall, the dendritic arbors appeared normal between $f_{S}(1) h^{17} /+$ heterozygous (Supplemental Fig. S5J, J') and $f_{S}(1) h^{17}$ hemizygous (Supplemental Fig. S5K, $\mathrm{K}^{\prime}$ ) larvae. In addition, the number of spike protrusions was identical between hemizygous and heterozygous mutants (Supplemental Fig. S5L). Therefore, Fsh-L does not appear to regulate class III dendrite morphology. In contrast, our data suggest the Fsh-S isoform regulates dendrite morphology.

The $f_{S}(1) h^{1112}$ mutation consists of a small deletion within the second bromodomain (BD2) of the $f_{s}(1) h$ gene, 


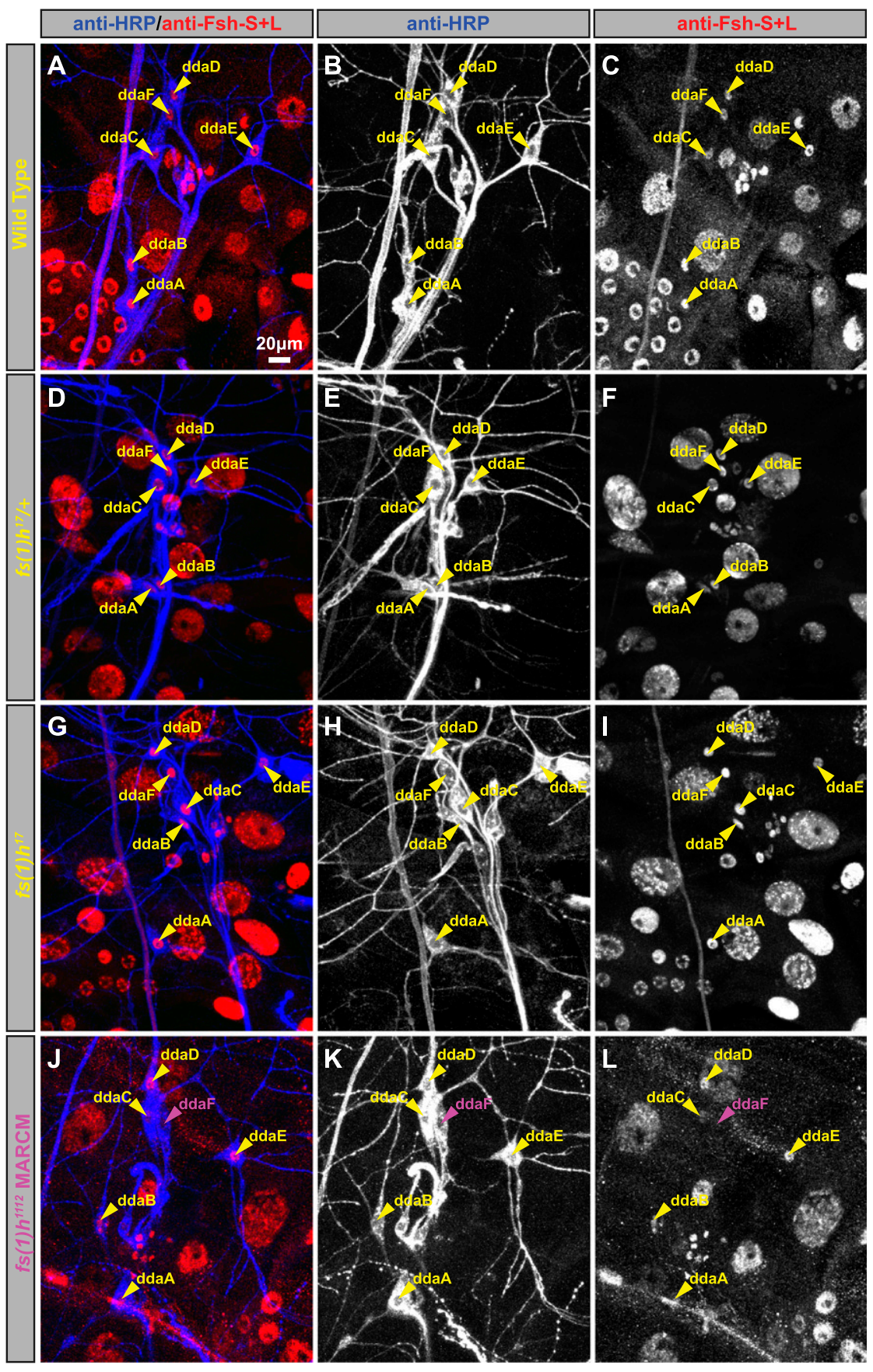

Figure 2. $f_{S}(1) h^{1112}$ causes a loss of Fsh protein expression. $(A-L)$ Immunohistochemical staining performed on third instar larval da sensory neurons using anti-HRP to label PNS neurons and anti-Fsh-S+L to visualize Fsh protein expression. The da neurons are indicated by name with yellow text and arrowheads marking the nucleus. The da neurons belong to different classes as follows: class I, ddaD and ddaE; class II, $\mathrm{ddaB}$; class III, ddaA and ddaF; and class IV, $\mathrm{ddaC}$. The genotype is denoted at the left of each row, and $f_{S}(1) h^{1112}$ mutant clones are indicated by pink text. Similar anti-Fsh-S signal is observed between wild-type $(A-C)$, $f_{S}(1) h^{17} /+(D-F)$, and $f_{S}(1) h^{17}(G-I)$ neurons. Comparing wild type $(A-C)$ with $f_{S}(1) h^{1112}$ $(J-L)$ indicates a loss of anti-Fsh-S+L signal in $f_{S}(1) h^{1112}$ clones compared with wild type. Bar,20 $\mu \mathrm{m}$.

causing a premature stop codon, which could lead to the generation of a truncated protein (Supplemental Fig. S3A). Thus, it is possible that the $f_{S}(1) h^{1112}$ allele creates a protein with a dominant-negative or gain-of-function effect. To assess whether any Fsh protein product is expressed in da neuron MARCM clones, we used the anti-Fsh-S+L antibody to analyze $f_{S}(1) h^{1112}$ da neuron clones, which recognizes an epitope (Supplemental Fig. S3A) preceding the premature stop codon. Comparing $f_{S}(1) h^{1112}$ with wild-type neurons from adjacent segments, we noticed a loss of staining in $f_{S}(1) h^{1112}$ class III clones (Fig. 2J-K). Thus, $f_{S}(1) h^{1112}$ appears to be a complete loss-of-function mutation.
The human fs(1)h homolog (huBRD2) substitutes for Fsh protein function in regulating dendrite morphology Since $f_{S}(1) h^{1112}$ causes a loss of anti-Fsh-S+L labeling (Fig. 2) and UAS-Fsh-S can partially rescue the reduction in dendrite arbor complexity (Fig. 1), we wanted to assess whether the human homolog can functionally substitute for Fsh-S. Comparing the protein sequence of Drosophila Fsh-S with its human homolog, BRD2 (huBRD2), indicates $69 \%$ similarity of FSH-S to huBRD2 and 54\% similarity of huBRD2 to Fsh-S (Supplemental Fig. S3B). Within domains of known functional importance (the two bromodomains 
[BD1 and BD2] and the extraterminal [ET] domain), there is $>70 \%$ similarity, indicating a high degree of conservation between Drosophila and human sequences. To test whether huBRD2 can rescue the dendrite phenotype of $f_{S}(1) h^{1112}$, we created a UAS-huBRD2 transgene to express the huBRD2 coding sequence in da sensory neuron clones. In $f_{S}(1) h^{1112}$ class III da neurons, the expression of $U A S$ $h u B R D 2$ significantly increased the number of dendritic spikes (Fig. 3C) compared with $f_{S}(1) h^{1112}$ alone (Fig. 3B) $\left[f_{S}(1) h^{1112} ; U A S-h u B R D 2 /+: ~ 119 \pm 10\right.$ spikes, $n=7$ cells vs. $f_{S}(1) h^{1112}: 31 \pm 3$ spikes, $n=9$ cells; $P<0.001$ ] (Fig. $\left.3 \mathrm{E}\right)$. Expression of $U A S$-huBRD2 in $f_{S}(1) h^{1112}$ class III da neurons did not alter the primary dendrite length $\left[f_{S}(1) h^{1112}\right.$; UAS-huBRD2/+: $2610 \mu \mathrm{m} \pm 270 \mu \mathrm{m}, n=7$ cells vs. $f_{S}(1) h^{1112}: 1951 \mu \mathrm{m} \pm 142 \mu \mathrm{m}, n=9$ cells; $\left.P=0.05\right]$ (Fig. 3F) or branch point number $\left[f_{S}(1) h^{1112}\right.$;UAS-huBRD2/+: $14 \pm 2$ branch points, $n=7$ cells vs. $f_{S}(1) h^{1112}: 10 \pm 1$ branch points, $n=9$ cells; $P=0.146$ ] (Fig. 3G). These findings reveal that huBRD2 partially rescues the $f_{S}(1) h^{1112}$ dendrite spike morphology phenotype.

$\mathrm{fs}(1) \mathrm{h}^{1112}$ affects initial establishment of dendritic arbor complexity

The reduction in dendrite complexity caused by $f_{S}(1) h^{1112}$ could be the result of a failure to establish dendritic branch complexity or a compromised maintenance of dendritic complexity during development. Thus far, we only assessed the dendritic morphology at the late third instar stage, corresponding to $\sim 100 \mathrm{~h}$ after egg laying (AEL). To determine whether $f_{S}(1) h^{1112}$ impairs establishment and/or maintenance of dendritic arbor complexity, we performed a time-course study comparing dendritic arbors of class III da neuron MARCM clones from wildtype control (Fig. 4A-C) and $f_{S}(1) h^{1112}$ (Fig. 4D-F) genotypes over three developmental stages $(48,72$, and $96 \mathrm{~h}$ AEL). Class III wild-type da neuron arbors continually increased their number of spikes (Fig. 4G) from 48 to $96 \mathrm{~h}$ AEL (48 h AEL: $69 \pm 4$ spikes, $n=4$ cells; 72 h AEL: $118 \pm$ 16 spikes, $n=5$ cells; 96 h AEL: $178 \pm 15$ spikes, $n=4$ cells) as their primary arbors lengthened (48 h AEL: 1100 $\mu \mathrm{m} \pm 77 \mu \mathrm{m}, n=4$ cells; $72 \mathrm{~h}$ AEL: $2119 \mu \mathrm{m} \pm 134 \mu \mathrm{m}$, $n=5$ cells; $96 \mathrm{~h}$ AEL: $2201 \mu \mathrm{m} \pm 65 \mu \mathrm{m}, n=4$ cells) (Fig. $4 \mathrm{H}$ ) with a modest increase of branch points (48 h AEL: $13 \pm 1$ branch points, $n=4$ cells; 72 h AEL: $14 \pm 1$ branch points, $n=5$ cells; $96 \mathrm{~h}$ AEL: $16 \pm 1$ branch points, $n=4$ cells) (Fig. 4I). In contrast, $f_{S}(1) h^{1112}$ class III da neuron arbors failed to proliferate spike protrusions $(48 \mathrm{~h}$ AEL: $51 \pm 4$ spikes, $n=4$ cells; 72 h AEL: $67 \pm 5$ spikes, $n=3$ cells; 96 h AEL: $57 \pm 5$ spikes, $n=5$ cells) (Fig. 4G). Their primary dendrites exhibited an increase in total

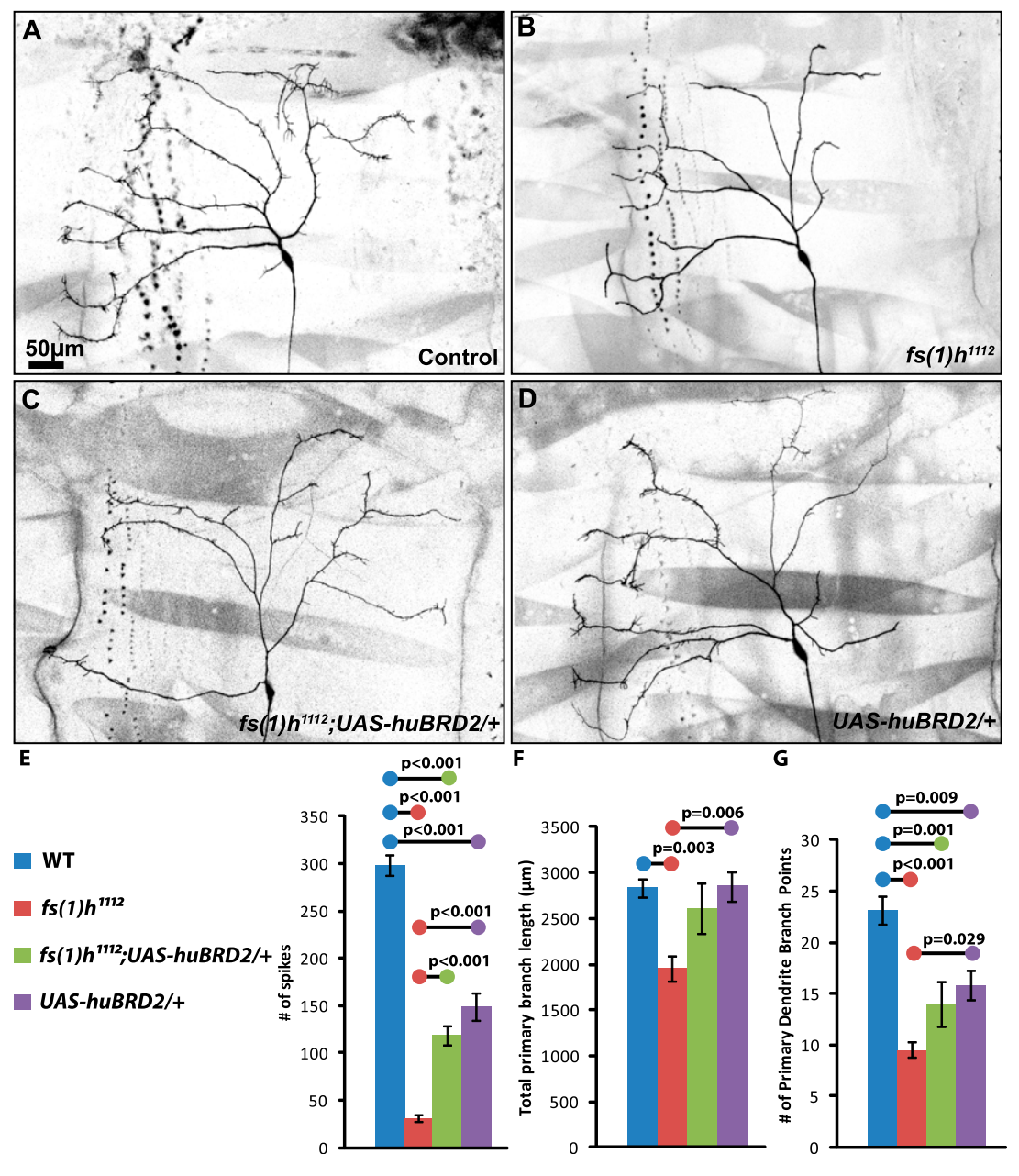

Figure 3. The $f_{S}(1) h^{1112}$ dendritic arbor phenotype can be rescued by the human $f_{S}(1) h$ homolog huBRD2. $(A-D)$ Dendritic arbors of class III da neuron MARCM clones in a control background $\left(y w, F R T^{19 A}\right.$; sop-FLP, $\left.G A L 4^{109(2) 80}, U A S-m C D 8-G F P /+\right)$ and crossed to various mutant and transgenic flies as indicated. Bar, $50 \mu \mathrm{m}$. The loss of spikes caused by $f_{S}(1) h^{1112}(B)$ is partially reversed by expression of $U A S-h u B R D 2(C)$ compared with the control $(A)$. Bar, $50 \mu \mathrm{m} .(E-G)$ Quantification of dendritic arbor morphology by number of spikes $(E)$, primary branch length $(F)$, and primary branch points $(G)$. (C) The loss of spikes in $f_{S}(1) h^{1112}$ clones is partially rescued by $U A S-h u B R D 2$. The $f_{S}(1) h^{1112}$-induced decrease in primary dendritic branch length $(F)$ and primary branch points $(G)$ is not rescued by $U A S-h u B R D 2$ expression. The values are reported as mean \pm SEM, and $P$-value statistical significance is reported by results of one-way ANOVA followed by post-hoc Tukey test. 


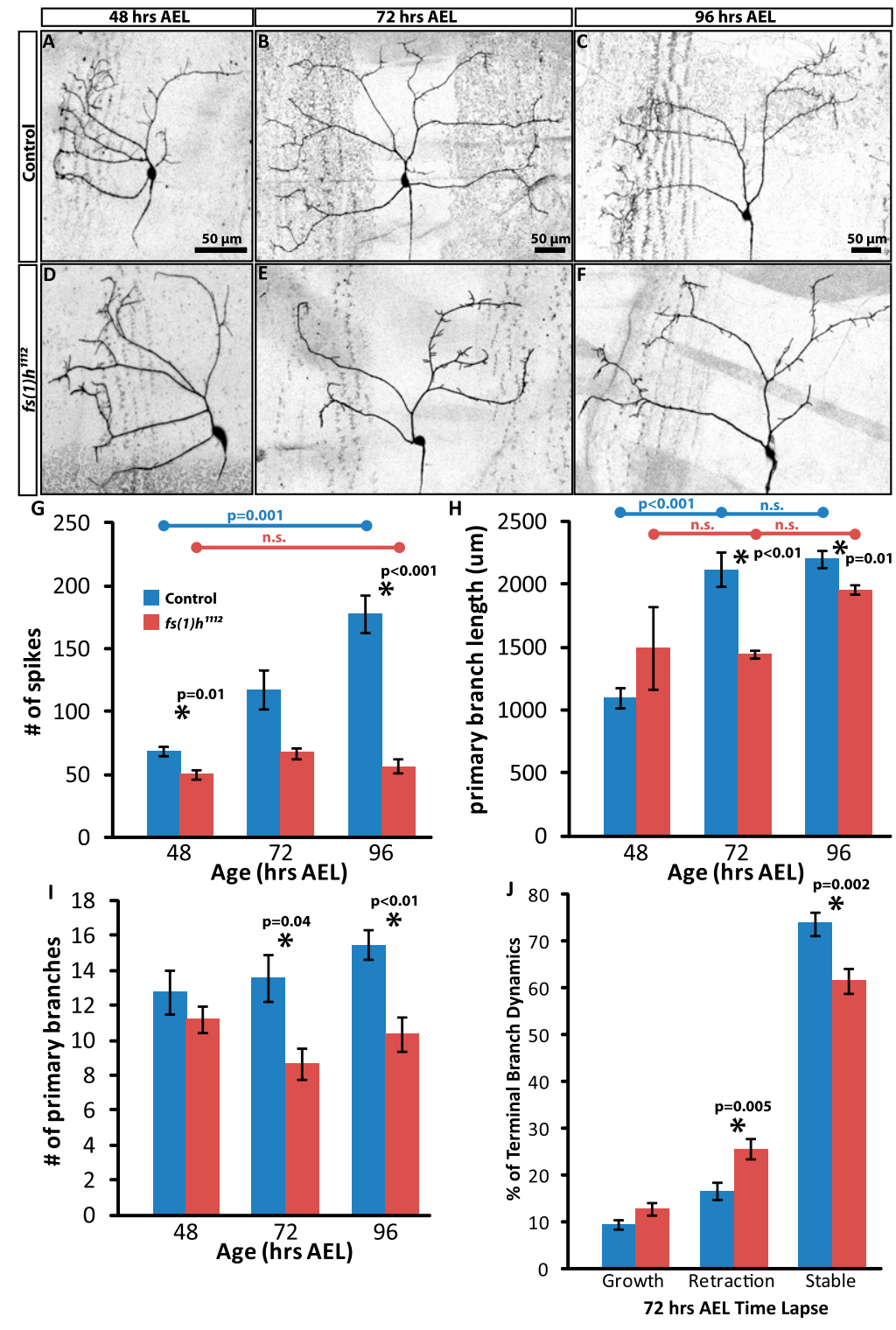

Figure 4. Initial elaboration of dendritic arbor is impaired by $f_{S}(1) h^{1112}$. $(A-F)$ Images of the dendritic arbors from control and $f_{S}(1) h^{112}$ MARCM class III da neuron clones at defined time points after egg laying (AEL). Bar, $50 \mu \mathrm{m}$. The overall shape and size of the dendritic arbors appear similar between control and $f_{S}(1) h^{1112}$ neurons, but the spike morphology is reduced in $f_{S}(1) h^{1112}$ neurons. $(G-I)$ Quantification and comparison of dendritic arbor morphology between developmental time points. Values are reported as mean \pm SEM, with statistical significance reported as results of one-way ANOVA and post-hoc Tukey test (colored horizontal bars) or Student's $t$-test $\left({ }^{\star}\right) .(G-I)$ The control neurons continually increase spike numbers, primary branch length, and primary branch points throughout development (blue bars). In contrast, $f_{S}(1) h^{1112}$ neurons ( $G-I$, red bars) fail to increase spike numbers and remain reduced compared with the control from the earliest time point examined $(G)$. The $f_{S}(1) h^{1112}$ mutant neurons do exhibit growth of primary branch length, although delayed from control $(H)$, and a decrease in primary branch length at later time points $(I)$. (J) Time-lapse imaging of $72 \mathrm{~h} \mathrm{AEL}$ MARCM clones over a 30-min imaging session. Terminal branch dynamics were classified as growing, retracting, or stable, and the overall percentage of each type of dynamic event was reported as mean \pm SEM. $f_{S}(1) h^{1112}$ neurons show an amount of growth similar to the control; however, the amount of retraction is increased, while the number of stable branches is decreased. dendrite length (48 h AEL: $1496 \mu \mathrm{m} \pm 333 \mu \mathrm{m}, n=4$ cells; $72 \mathrm{~h}$ AEL: $1448 \mu \mathrm{m} \pm 29 \mu \mathrm{m}, n=3$ cells; 96 h AEL: 1959 $\mu \mathrm{m} \pm 37 \mu \mathrm{m}, n=5$ cells), although this increase was delayed compared with wild-type clones (Fig. 4H). Since there is some increase in dendritic length without an increase in higher-order dendritic spikes, our data suggest that $f_{S}(1) h^{1112}$ impairs the initial establishment of dendritic arbor complexity.

A change in finer branch dynamics could explain the reduced dendritic complexity phenotype. To test this hypothesis, we performed acute time-lapse imaging of MARCM clones at $72 \mathrm{~h}$ AEL over a 30 -min period. The terminal branch dynamics were classified as growing, retracting, or stable at each time point. We observed no change in the amount of class III da neuron spike growth between the control and $f_{S}(1) h^{1112}$ [wild type: $9.5 \% \pm 1.0 \% ; f_{S}(1) h^{1112}: 12.8 \% \pm 1.4 \% ; P=0.06$ ] (Fig. $\left.4 \mathrm{~J}\right)$. However, the amount of retracting spikes increased in $f_{S}(1) h^{1112}$ neurons [wild type: $16.7 \% \pm 1.9 \% ; f_{S}(1) h^{1112}$ :
$25.7 \% \pm 2.3 \% ; P=0.005$ ] (Fig. 4J), while the amount of stable spikes was reduced [wild type: $73.8 \% \pm 2.5 \%$; $\left.f_{S}(1) h^{1112}: 61.5 \% \pm 2.6 \% ; P=0.002\right]$ (Fig. 4J). Therefore, the spikes of class III da neurons lacking Fsh-S show a greater tendency to retract and reduced stability, which cause a failure to establish higher-order dendritic spikes.

\section{$\mathrm{fs}(1) \mathrm{h}$ interacts with genetic programs regulating dendritic development}

We next wanted to explore the mechanism of how $f_{S}(1) h$ regulates dendritic morphology. One idea posits that neuron subtype-specific morphology arises from expression of specific combinations of molecules (Jan and Jan 2010). Previous studies have identified a variety of proteins expressed in da neurons that regulate dendrite morphology (Grueber et al. 2003; Li et al. 2004; Sugimura et al. 2004; Kim et al. 2006; Hattori et al. 2007; Jinushi-Nakao et al. 2007). Therefore, it is possible that $f_{S}(1) h$ could regulate 
the combinatorial expression of molecular factors governing dendrite development. In particular, the transcription factor encoded by $c t$ regulates the dendritic spikes of class III da neuron arbors. To test this hypothesis, we first examined the expression of Ct protein in $f_{S}(1) h^{1112}$ da neuron clones. We observed a loss of anti-Ct staining intensity in class III da neurons (Fig. 5A-F). Next, we tested whether expression of UAS-Ct could rescue the $f_{S}(1) h^{1112}$ dendrite phenotype. Although expression of $U A S-C t$ in $f_{S}(1) h^{1112}$ clones seemed to mildly affect induction of spike formation (Fig. 5G,g,g $g^{\prime}$ ), this effect was variable throughout the dendritic arbor, and we did not observe a statistically significant increase in spike number $\left[f_{s}(1) h^{1112} ; U A S-C t /+: 48 \pm 4\right.$ spikes, $n=7$ cells vs. $f_{S}(1) h^{1112}: 36 \pm 4$ spikes, $n=9$ cells; $P=0.989$ ] (Fig. 5I). Since $f_{S}(1) h$ binds acetylated histones to alter transcription, it is possible that expression of the UAS-Ct transgene was altered in $f_{S}(1) h^{1112}$ mutants. To address this concern, we examined the expression of Ct protein with immunohistochemistry in $f_{S}(1) h^{1112}$ class III da neuron clones. In this experiment, we observed a high expression of Ct protein (Supplemental Fig. S6). Thus, we confirmed that $\mathrm{Ct}$ protein was expressed by UAS-Ct, and therefore, rescuing Ct protein expression in $f_{s}(1) h^{1112} \mathrm{mu}-$ tant neurons cannot rescue the loss of class III dendritic spike morphology.

The transcription factor $\mathrm{Ct}$ regulates class-specific dendrite morphology and is thought to act by regulating a cascade of molecular factors. Therefore, $f_{s}(1) h$ may regulate some of these downstream components, which could explain why expression of UAS-Ct did not rescue the class III dendrite phenotype caused by loss of Fsh. Ectopic expression of UAS-Ct in class I da neurons causes extensive overbranching of their normally simple arbors as well as the formation of ectopic dendritic spikes similar to those observed in class III da neurons (Grueber et al. 2003). This paradigm can be used to probe whether $f_{S}(1) h^{1112}$ alters Ct-induced branching and spike formation. As previously shown (Grueber et al. 2003), expressing UAS-Ct in wild-type class I da neurons (Fig. 6B) caused an $86 \%$ increase in total dendrite length (UAS$C t /+: 3710 \mu \mathrm{m} \pm 265 \mu \mathrm{m}, n=4$ cells vs. wild type: 1996 $\mu \mathrm{m} \pm 71 \mu \mathrm{m}, n=5$ cells; $P<0.001$ ) (Fig. $6 \mathrm{H}$ ), a $287 \%$ increase in branch point number (UAS-Ct/+: $116 \pm 13$ branch points, $n=4$ cells vs. wild type: $30 \pm 1$ branch points, $n=5$ cells; $P<0.001$ ) (Fig. 6G), and the formation of dendritic spike morphology (Fig. 6B). In $f_{s}(1) h^{1112}$ class I da neuron clones, this induction of overbranching by UAS-Ct expression was abolished (Fig. 6C) with no increase in dendrite length $\left[f_{S}(1) h^{1112} ; U A S-C t /+\right.$ : 1930 $\mu \mathrm{m} \pm 23 \mu \mathrm{m}, n=4$ cells vs. wild type: $1996 \mu \mathrm{m} \pm 71$ $\mu \mathrm{m}, n=5$ cells; $P=0.985]$ (Fig. $6 \mathrm{H}$ ), branch point number $\left[f_{S}(1) h^{1112} ; U A S-C t /+: 43 \pm 6\right.$ branch points, $n=4$ cells vs. wild type: $30 \pm 1$ branch points, $n=5$ cells; $P=0.533$ ] (Fig. $6 F$ ), or the emergence of spikes (Fig. 6C). Finally, we tested whether UAS-Fsh-S expression can induce an overbranching phenotype in class I da neurons (Fig. 6D). This did not occur, and dendritic arbors of $f_{S}(1) h^{1112}$ mutant class I da neurons expressing UAS-Fsh-S appeared identical to wild-type class I arbors with similar dendritic length (UAS-Fsh-S/+: $2265 \mu \mathrm{m} \pm 95 \mu \mathrm{m}, n=4$ cells vs. wild type: $1996 \mu \mathrm{m} \pm 71 \mu \mathrm{m}, n=5$ cells; $P=0.517$ ) (Fig. $6 \mathrm{H}$ ), branch point number (UAS-Fsh-S/+: $29 \pm 2$ branch points, $n=4$ cells vs. wild type: $30 \pm 1$ branch points, $n=5$ cells; $P=0.999$ ) (Fig. $6 \mathrm{G}$ ), and lack of spikes. Thus, $f_{S}(1) h$ is necessary for UAS-Ct-induced overbranching and spike formation in class I da neuron arbors, but UAS-Fsh-S overexpression does not cause any change in class I dendritic morphology.

The spike morphology of class III da neuron dendritic arbors is known to be F-actin-rich (Grueber et al. 2002) and can be altered by modulating the activity of the GTPase Rac1 (Andersen et al. 2005; Tsubouchi et al. 2012). As mentioned earlier, ectopic expression of Ct causes spike formation in class I da neuron dendritic arbors (Grueber et al. 2003). Ectopic Rac1 expression causes increased branching in class I da neuron arbors, which is similar but not identical to class III da neuron spikes. Interestingly, coexpression of $\mathrm{Ct}$ and Racl synergistically enhances the formation of spike morphology in class I da neurons (Jinushi-Nakao et al. 2007). Therefore, Ct and Racl function in concert to specify the dendritic arbor shape of class III da neurons. For this reason, we wanted to test whether the loss of dendritic spikes in $f_{S}(1) h^{1112}$ mutant class III da neurons could be rescued by UAS-Rac1. Indeed, we observed a significant $157 \%$ of control) increase in spike number after UAS-Rac1 expression in $f_{S}(1) h^{1112}$ mutant class III da neuron clones $\left[f_{S}(1) h^{1112} ; U A S-R a c 1 /+: 149 \pm 16\right.$ spikes, $n=9$ cells vs. $f_{S}(1) h^{1112}: 36 \pm 4$ spikes, $n=9$ cells; $P<0.001$ ] (Fig. $5 \mathrm{H}, \mathrm{h}, \mathrm{h}^{\prime}, \mathrm{I}, \mathrm{l}$. This rescue was partial but similar to that achieved with UAS-Fsh-S expression in $f_{S}(1) h^{1112}$ class III da neurons (Fig. 1). Moreover, in agreement with a previous report (Jinushi-Nakao et al. 2007), when we ectopically expressed UAS-Rac1 in class I da neurons (Fig. 6E), we observed a significant increase in branch points (UASRac1/+: $79 \pm 10$ branch points, $n=7$ cells vs. wild type: $30 \pm 1$ branch points, $n=5$ cells; $P=0.001$ ) (Fig. 6G), although to a much less degree than observed with UAS$C t$ (Fig. 6G,H). In contrast to ectopic UAS-Ct, expression of UAS-Rac1 did not significantly alter total dendrite length (UAS-Rac1/+: $2394 \mu \mathrm{m} \pm 94 \mu \mathrm{m}, n=7$ cells vs. wild type: $1996 \mu \mathrm{m} \pm 71 \mu \mathrm{m}, n=5$ cells; $P=0.118$ ) (Fig. 6H). Finally, we tested whether the UAS-Rac1induced increase in class I da neuron dendritic branching was altered in $f_{s}(1) h^{1112}$ mutants (Fig. 6F). Unlike the abolition of $C t$-induced overbranching by $f_{S}(1) h^{1112}$, the Rac1-induced overbranching was unaltered $\left[f_{S}(1) h^{1112}\right.$; UAS-Rac1/+: $89 \pm 5$ branch points, $n=8$ cells vs. UASRac1/+: $79 \pm 10$ branch points, $n=7$ cells; $P=0.886$ ] (Fig. 6G). Thus, enhanced Racl expression can partially rescue the $f_{s}(1) h^{1112}$ class III da neuron dendritic phenotype, and $f_{S}(1) h$ is necessary for Ct-induced dendritic branching but not Rac1-induced branching.

Because Ct is also expressed in class II and IV da neurons, we also assessed whether the $f_{s}(1) h^{1112}$ mutation altered $\mathrm{Ct}$ expression in all da neuron subtypes. Similar to our observations in $f_{S}(1) h^{1112}$ mutant class III da neurons, Ct expression was lost in both class II (Supplemental Fig. S7B,E,H) and class IV (Supplemental 


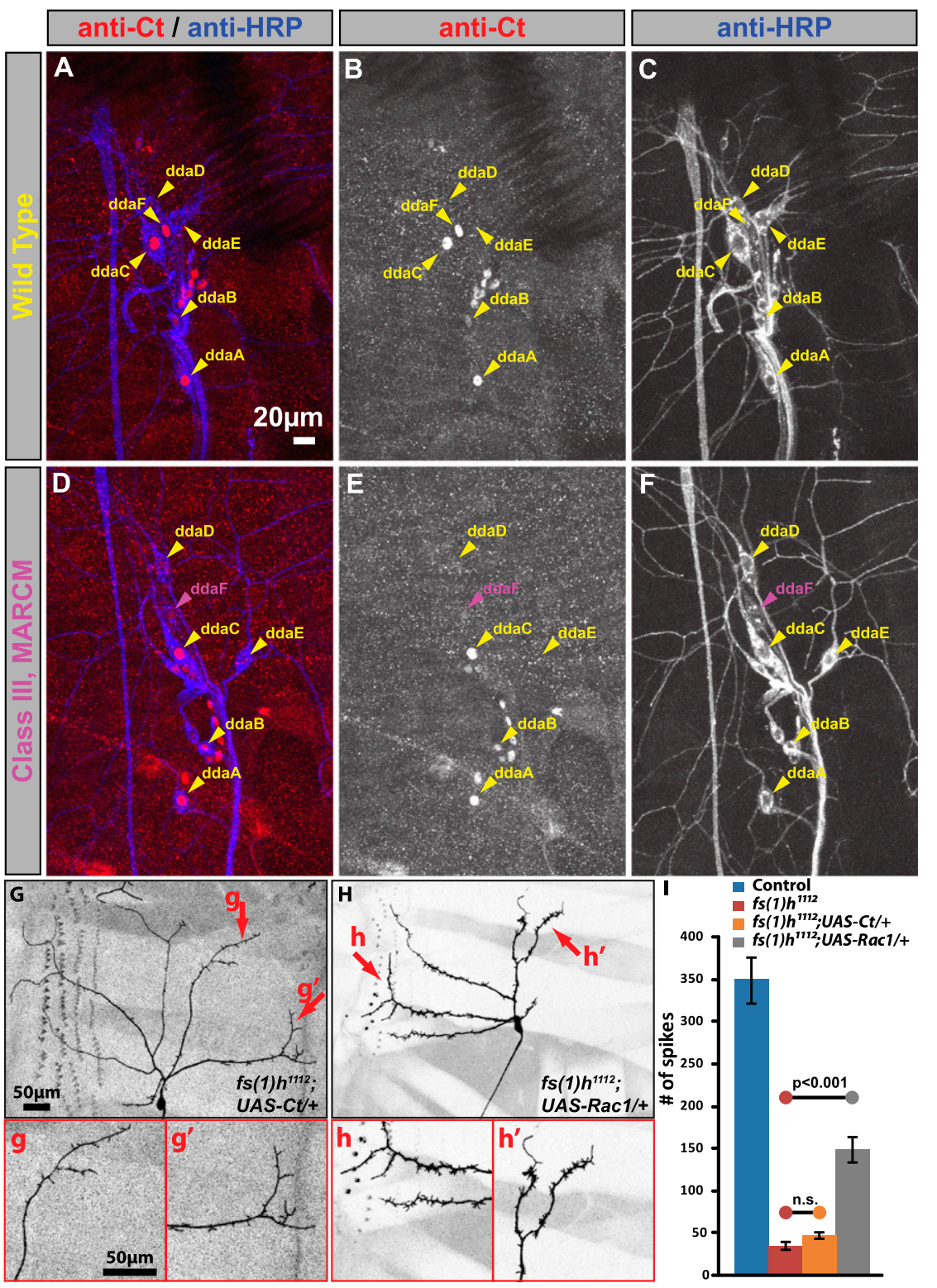

Fig. S7C,F,I) mutant da neurons. Since Ct is normally not expressed in class I da neurons, we asked whether the $f_{S}(1) h^{1112}$ mutation caused misexpression of Ct. This was not the case, and we did not detect any Ct expression in $f_{S}(1) h^{1112}$ mutant class I da neurons (Supplemental Fig. $\mathrm{S} 7 \mathrm{~A}, \mathrm{D}, \mathrm{G})$. Therefore, $f_{S}(1) h$ regulates the expression of $\mathrm{Ct}$ in the da neurons that normally express $\mathrm{Ct}$.

Because overexpression of Fsh-S also caused a dendrite phenotype similar to $f_{S}(1) h^{1112}$ mutant class III da neurons (Fig. 1), we tested whether overexpression of Fsh-S affected Ct expression and/or Ct-induced branching/spike formation. First, we examined the pattern of anti-Ct antibody labeling in larvae expressing UAS-Fsh-S under the control of a pan-da neuron GAL4 driver $\left(G A L 4^{109(2) 80}\right)$. We did not observe a change in anti-Ct signal between control (Supplemental Fig. S8A,C,E) and UAS-Fsh-S-expressing (Supplemental Fig. S8B,D,F) da neurons. These data suggest that Fsh-S overexpression does not lead to decreased Ct
Figure 5. $f_{S}(1) h^{1112}$ neurons do not express Ct protein, and UAS-Rac1 but not UAS-Ct expression rescues the dendrite defects. $(A-$ $F$ ) Immunohistochemical staining with anti-Ct (clp2 epitope) and anti-HRP in da sensory neurons. The da neurons belong to different classes as follows: class $\mathrm{I}, \mathrm{ddaD}$ and ddaE; class II, ddaB; class III, ddaA and ddaF; and class IV, ddaC. Wild-type neurons (labeled in yellow) and $f_{S}(1) h^{1112}$ class III MARCM clones (labeled in pink) are indicated, and while anti-Ct signal is apparent in wild-type class III neurons $(A-C)$, it is lost in $f_{S}(1) h^{1112}$ clones $(D-F)$. Bar, $20 \mu \mathrm{m}$. $(G, H)$ Images of $f_{S}(1) h^{1112}$ MARCM clones expressing UAS-Ct or UAS-Rac1, with magnified inset of terminal dendrite morphology (shown in $g, g^{\prime}, h, h^{\prime}$ ). Bar, $50 \mu \mathrm{m}$. With UAS-Ct rescue, spike morphology is apparent but inconsistent throughout the dendritic arbor. In contrast, UAS-Rac1 rescues spike formation in $f_{S}(1) h^{1112}$ mutant neurons. (I) Quantification of spike morphology, which indicates no rescue of the $f_{S}(1) h^{1112}$-induced decrease with UAS-Ct expression but a significant rescue with UAS-Rac1 expression. Values are reported as mean \pm SEM, with statistical significance reported as results of one-way ANOVA and post-hoc Tukey test. expression as observed in $f_{S}(1) h^{1112}$ mutants and that Fsh-S overexpression does not induce misexpression of $\mathrm{Ct}$ in da neurons that normally do not express $\mathrm{Ct}$.

Second, we assayed whether Fsh-S overexpression can affect Ct-induced branching/spike formation in class $1 \mathrm{da}$ neurons. Using the class I da neuron GAL4 $4^{2-21}$ driver (Grueber et al. 2003), we overexpressed UAS-Fsh-S along with UAS-Ct (Supplemental Fig. S9). As observed previously (Grueber et al. 2003), UAS-Ct expression in class I da neurons caused an increase in total dendrite length (control: $1733 \mu \mathrm{m} \pm 56 \mu \mathrm{m}, n=10$ cells vs. UAS-Ct/+: $4149 \mu \mathrm{m} \pm 143 \mu \mathrm{m}, n=9$ cells; $P<0.001$ ) (Supplemental Fig. S9E) and branch points (control: $31 \pm 2$ branch points, $n=10$ cells vs. UAS-Ct/+: $149 \pm 8$ branch points, $n=9$ cells; $P<0.001$ ) (Supplemental Fig. S9F), with the production of class III da neuron-like morphology (Supplemental Fig. S9B, $\left.b^{\prime}\right)$, which we also observed in our MARCM experiments (Fig. 6). Similar to our MARCM 
observations (Fig. 6), UAS-Fsh-S expression in class I da neurons caused no alteration in total dendrite length [control: $1733 \mu \mathrm{m} \pm 56 \mu \mathrm{m}, n=10$ cells vs. UAS-Fsh$S /+: 1765 \mu \mathrm{m} \pm 37 \mu \mathrm{m}, n=9$ cells; $P=0.995$ ] (Supplemental Fig. S9E) or branch points (control: $31 \pm 2$ branch points, $n=10$ cells vs. UAS-Fsh-S/+: $26 \pm 1$ branch points, $n=9$ cells; $P=0.956$ ) (Supplemental Fig. S9F). When UASFsh-S and UAS-Ct were overexpressed together in class I da neurons, we still observed the production of class III da neuron spike morphology (Supplemental Fig. S9D, d') and an increase in total dendrite length (control: $1733 \mu \mathrm{m} \pm$ $56 \mu \mathrm{m}, n=10$ cells vs. UAS-Ct/+;UAS-Fsh-S/+: $3588 \mu \mathrm{m} \pm$ $121 \mu \mathrm{m}, n=10$ cells; $P<0.001$ ) (Supplemental Fig. S9E) and branch points (control: $31 \pm 2$ branch points, $n=10$ cells vs. UAS-Ct/+;UAS-Fsh-S/+: $109 \pm 9$ branch points,

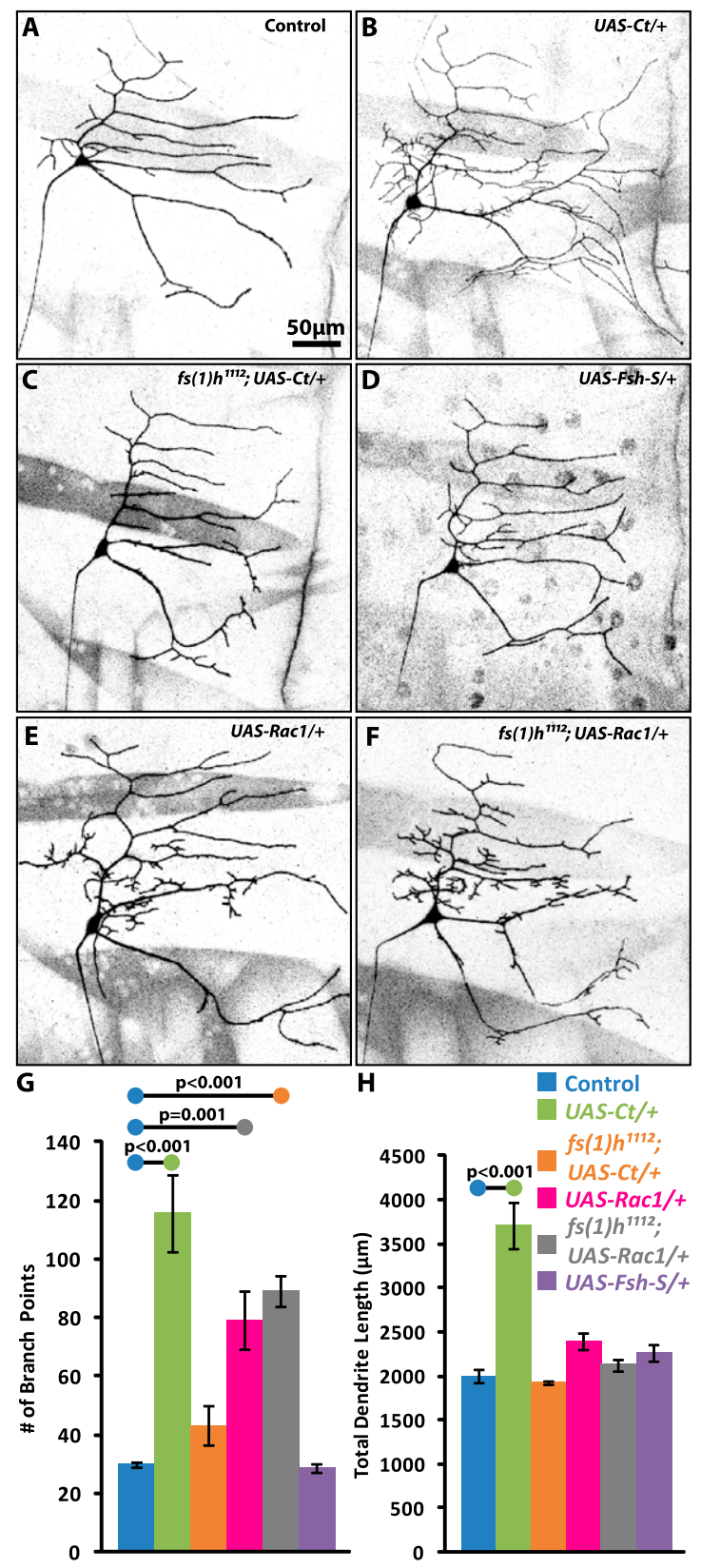

$n=10$ cells; $P<0.001$ ) (Supplemental Fig. S9F). However, the effects were significantly less than UAS-Ct expression alone for both total dendrite length $(U A S-C t /+: 4149 \mu \mathrm{m} \pm$ $143 \mu \mathrm{m}, n=9$ cells vs. UAS-Ct/+;UAS-Fsh-S/+: $3588 \mu \mathrm{m} \pm$ $121 \mu \mathrm{m}, n=10$ cells; $P=0.002$ ) (Supplemental Fig. S9E) and branch points $(U A S-C t /+: 149 \pm 8$ branch points, $n=9$ cells vs. UAS-Ct/+;UAS-Fsh-S/+: $109 \pm 9$ branch points, $n=10$ cells; $P<0.001$ ) (Supplemental Fig. S9F). These data suggest that Fsh-S overexpression attenuates UAS-Ct-induced branching, which could explain why UAS-Fsh-S expression causes only a partial rescue of the $f_{S}(1) h^{1112}$ mutant phenotype and also why UAS-Fsh-S overexpression causes a dendrite phenotype similar to $f_{S}(1) h^{1112}$.

Having found dendritic phenotypes in all da neuron subtypes, we further examined whether $f_{S}(1) h^{1112}$ altered the expression of other transcription factors known to regulate the subtype-specific dendritic morphology of da neurons. Specifically, we used antibodies against the class I da neuron-specific transcription factor encoded by $a b$ (Li et al. 2004) and the class IV-specific kn/col (Crozatier and Vincent 2008). Interestingly, we observed a loss of both $\mathrm{Ab}$ and $\mathrm{Kn} / \mathrm{Col}$ in $f_{S}(1) h^{1112}$ mutant class I (Supplemental Fig. S10) and class IV (Supplemental Fig. S11) da neurons, respectively. Collectively, these data suggest that $f_{S}(1) h$ regulates subtype-specific expression of transcription factors. It affects not only the expression of $c t$ across multiple neural subtypes but also transcription factors in pathways acting in parallel to $c t$, such as $a b$ and $\mathrm{kn} / \mathrm{col}$.

\section{$\mathrm{fs}(1) \mathrm{h}^{1112}$ impairs the mechanosensory response of da sensory neurons}

A recent study discovered that NompC mediates gentle touch behavior and mechanosensitivity in class III da neurons (Yan et al. 2013). Since the $f_{s}(1) h^{1112}$ mutation alters the morphology of class III da neurons, we tested whether $f_{S}(1) h$ modulates their touch sensitivity. Since $f_{s}(1) h^{1112}$ mutants are lethal, we could not use whole-

Figure 6. UAS-Ct-induced overbranching in class I (ddaE) da neurons is blocked by $f_{S}(1) h^{1112}$, while UAS-Rac1-induced branching is unaffected. $(A-D)$ Images of dendrite morphology from class I (ddaE) MARCM clones in control background (yw, $F R T^{19 A}$; sop-FLP, GAL4 $\left.{ }^{109(2) 80}, U A S-m C D 8-G F P /+\right)$ with the indicated genotypes. Bar, $50 \mu \mathrm{m}$. Excess branching and spike formation are readily apparent with UAS-Ct expression $(B)$, but this overbranching is not observed in a $f_{S}(1) h^{1112}$ background $(C) .(D)$ Overexpression of Fsh-S protein does not induce overbranching. Excess branching occurs with UAS-Rac1 expression $(E)$, and this overbranching is unaffected in a $f_{S}(1) h^{1112}$ background $(F)$. $(G, H)$ Quantification of dendrite morphology by total dendritic length $(G)$ and number of branch points $(H)$. Expression of UAS-Ct causes an increase in both length and branching compared with the control. This increase does not occur in a $f_{S}(1) h^{1112}$ background. Expression of UAS-Rac1 only causes an increase in branching, and this also occurs in a $f_{S}(1) h^{1112}$ background. Finally, there is no change with overexpression of UAS-Fsh-S. Values are reported as mean \pm SEM, with statistical significance reported as results of one-way ANOVA and post-hoc Tukey test. 
animal mutants. Instead, we used $G A L 4^{19-12}$ to express a UAS-RNAi construct (v51227) against $f_{S}(1) h$ in class III da neurons to knock down $f_{S}(1) h$ expression selectively in these neurons. As a control for the effectiveness of the RNAi construct, we tested whether UAS-RNAi expression altered class III da neuron spike morphology. Indeed, RNAi-mediated knockdown of $f_{S}(1) h$ caused a significant decrease in spike number (v51227 RNAi: $151 \pm 9$ spikes, $n=6$ cells vs. wild type: $260 \pm 9$ spikes, $n=3$ cells; $P<0.001$ ) (Fig. 7A). Using a separate RNAi line (v108662), we observed a similar decrease in spike number (v108662 RNAi: $151 \pm 8$ spikes, $n=6$ cells vs. wild type: $260 \pm 9$ spikes, $n=3$ cells; $P<0.001$ ) (Fig. 7A). This decrease was enhanced in a genetic background containing a heterozygous deletion mutant [ $\mathrm{f}(\mathrm{x}) \mathrm{c} 128]$ of $f_{S}(1) h[D f(x) c 128 /+; v 108662$ RNAi: $96 \pm 9, n=6$ cells vs. wild type: $260 \pm 9, n=3$ cells; $P<0.001$ ] (Fig. 7A). Thus, RNAi-mediated knockdown of $f_{S}(1) h$ expression reduced spike morphology in class III da neuron dendritic arbors.

We next tested whether animals expressing RNAi constructs against $f_{S}(1) h$ exhibited any defects in gentle touch behavior. A previous genetic screen used a gentle touch assay to identify touch-insensitive mutants (Kernan et al. 1994), and this assay was used to determine that class III da neurons mediate this gentle touch behavior (Yan et al. 2013). Using the same assay, we observed a significant $(P=0.02)$ reduction in touch response score in animals expressing $f_{S}(1) h$ RNAi (Fig. 7B). To determine whether the $f_{S}(1) h$ RNAi affects mechanosensation of class III da neurons as opposed to subsequent signaling in the CNS, we performed extracellular recordings from class III da neurons while delivering a touch stimulus of varying intensity. Control class III da neurons responded with a progressively higher frequency of action potentials (APs) as the touch stimulus intensity increased (Fig. 7C,D). Although class III da neurons expressing $f_{S}(1) h$ RNAi also showed a dose-dependent response with increasing stimulus intensity (Fig. 7C), this response was significantly reduced compared with control neurons (Fig. 7C,D). Therefore, knockdown of $f_{S}(1) h$ expression impairs the response of class III da neurons to touch.

Since NompC is the channel mediating the mechanosensitive response of class III da neurons, one possible explanation for their defective response is a loss or reduction of NompC expression. To address this possibility, we performed immunohistochemistry with an antiNompC antibody in $f_{S}(1) h^{1112}$ class III clones. In both wild-type and $f_{S}(1) h^{1112}$ neurons, we observed antiNompC signal (Supplemental Fig. S12) similar to a previous study (Yan et al. 2013). Therefore, the impaired sensory response of class III da neurons expressing $f_{S}(1) h$ RNAi is not due to a loss of NompC expression.

To test whether the defective response to gentle touch reflects an alteration of sensory neuron axon structure, we examined the axon terminals of $f_{S}(1) h^{1112}$ mutant class III da neurons. The axon terminals appeared similar between wild-type control (Fig. 7E) and $f_{S}(1) h^{1112}$ mutant (Fig. 7F) class III da neurons. In addition, we did not find a significant change in axon terminal length $\left[f_{S}(1) h^{1112}\right.$ :
$92 \mu \mathrm{m} \pm 13 \mu \mathrm{m}, n=13$ cells vs. wild type: $108 \mu \mathrm{m} \pm 11$ $\mu \mathrm{m}, n=13$ cells; $P=0.353$ ] (Fig. 7G). Therefore, the reduction of gentle touch response by $f_{S}(1) h^{1112}$ is not due to impaired axon terminal morphology. Rather, the $f_{S}(1) h^{1112}$ mutation altered dendrite morphology of class III da neurons without affecting NompC expression or axon morphology.

Recently, it was discovered that ectopic UAS-Ct expression in class I da neurons induces expression of NompC, which is normally expressed in class III, but not class I, da neurons. Since we observed a loss of $\mathrm{Ct}$ expression but normal expression of NompC in $f_{S}(1) h^{1112}$ mutant class III da neurons (Supplemental Fig. S12), we wanted to test whether $f_{S}(1) h$ is required for the $C t$-induced expression of NompC in class I da neurons. When we performed anti-NompC immunostaining of class I da neuron $f_{S}(1) h^{1112}$ MARCM clones expressing $U A S-C t$, we observed expression of NompC (Supplemental Fig. S13C,D), which was not observed in wild-type class I da neurons (Supplemental Fig. S13A,B), even though the normal class III expression pattern of NompC was observed in the same dorsal da neuron cluster. We also observed $U A S$-Ct-induced NompC expression from class I da neurons in a $f_{S}(1) h^{1112}$ mutant background (Supplemental Fig. S13E,F). Therefore, $f_{S}(1) h^{1112}$ does not alter $C t$-induced NompC expression, which is interesting because $f_{S}(1) h^{1112}$ does block the $C t$-induced overbranching (Fig. 6). These data suggest that $f_{S}(1) h$ regulates genes involved in specifying dendritic arbor morphology but not ion channel expression.

\section{Discussion}

\section{fs $(1)$ h regulates dendritic arbor complexity}

In the current study, we examined the role of $f_{S}(1) h$ in dendritic development. We analyzed the effect of a lossof-function allele $\left[f_{S}(1) h^{1112}\right]$ on the morphology of class III da sensory neurons in the Drosophila PNS. Overall, $f_{S}(1) h^{1112}$ causes a reduction in dendritic arbor complexity, most notably in the finer, higher-order branches. We were able to partially rescue this reduced morphological complexity by reintroducing Drosophila Fsh-S or the human homolog (huBRD2) proteins. Furthermore, we found one aspect of the genetic mechanism of action for $f_{S}(1) h$ to be regulating the expression of $c t$ (and possibly other genes in the pathway) in multiple da neuron subtypes as well as subtype-specific transcription factors, such as Abrupt for class I and Knot/Collier for class IV da neurons, which in turn affect subtype-specific dendrite development. Our data show that $f_{S}(1) h$ regulates genetic pathways controlling dendritic arbor development but does not specify which ion channels are expressed. Finally, our results suggest that the subtype-specific spike morphology is important for an optimal response to relevant sensory stimuli in the mechanosensitive class III da neurons.

The development of a dendritic arbor involves multiple steps (Whitford et al. 2002; Corty et al. 2009; Puram and Bonni 2013) beginning with differentiation, where a neuronal precursor acquires a neural fate. Next, neurites 
begin to extend, and a neuron becomes polarized as neurites are designated as axon or dendrite. The immature axons and dendrites continue to grow as the neuron and the nervous system develop. Initially, the dendritic arbors are simple, with only a few primary dendrites, but as development progresses, the number of branches and overall arbor size increase. The terminal dendrite branches are dynamic throughout development, exhibiting growth, retraction, or stability. In addition, as the animal body size increases, the dendritic field area increases, and therefore a dendritic arbor must scale accordingly. Thus, dendritic development involves a complex plethora of processes, and dendritic morphology could be altered by affecting any of these processes. For instance, if the balance of dendrite dynamics is shifted

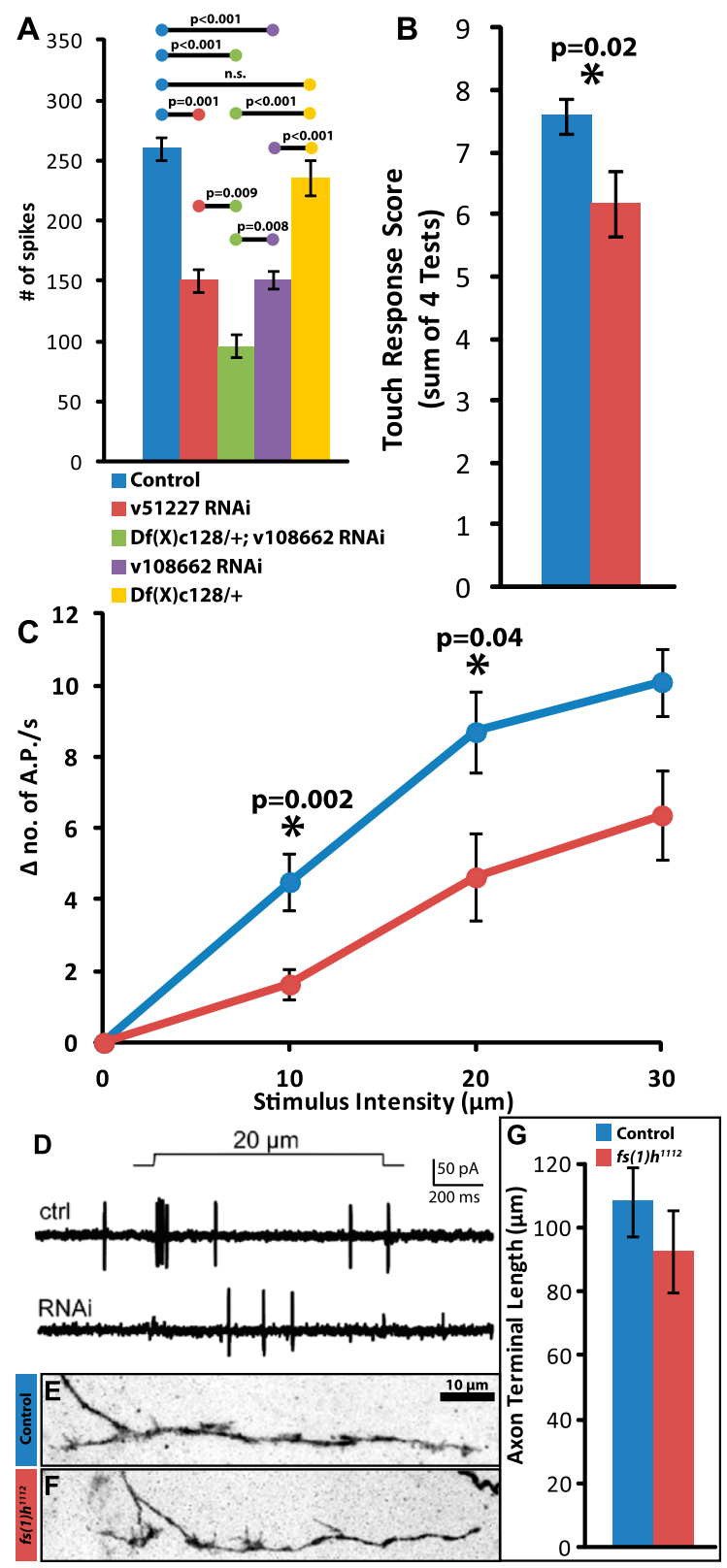

such that retraction is greater than growth, then dendritic branching will become reduced over time. This appears to be the case in $f_{S}(1) h^{1112}$ mutants, since we observed an increase in retracting branches with no change in growth as well as a decrease in the proportion of stable branches (Fig. 4) in dendritic arbors of $f_{S}(1) h^{1112}$ mutant da neuron clones compared with wild-type clones. Alternatively, if scaling of the dendritic arbor is affected, the size of the dendritic arbor will become disproportionately small as the body size of the animal increases throughout development (Parrish et al. 2009). This does not seem to occur in $f_{S}(1) h^{1112}$ mutants because the primary dendrites of $f_{S}(1) h^{1112}$ arbors exhibited growth throughout development, although at a delayed rate (Fig. 4). Instead, the number of spikes in class III da neuron arbors was reduced early in development and remained reduced throughout development (Fig. 4), probably due to the increased amount of dendritic branch retraction and reduced stability. Since the primary dendritic branches were not affected to a large degree by loss of $f_{S}(1) h$ function, we conclude that the major role of $f_{S}(1) h$ in dendritic development is to regulate dendritic complexity at the level of higher-order dendritic spikes. Moreover, our data suggest that $f_{S}(1) h$ affects dendritic arbor complexity by modulating the dynamics of terminal dendritic branches.

\section{$\mathrm{fs}(1) \mathrm{h}$ is necessary for Ct expression and Ct-induced spike formation}

In the da neurons, many molecules are known to regulate dendrite morphology. In particular, $\mathrm{Ct}, \mathrm{Ss}, \mathrm{Ab}$, and $\mathrm{Kn}$ have been shown to regulate subtype-specific morphology of the four classes of da sensory neurons, and these proteins act in parallel genetic pathways /Grueber et al.

Figure 7. Altering the number of spikes correlates with a reduced mechanosensitive response. (A) Quantification of spike morphology in class III da neurons expressing $f_{S}(1) h$ RNAi in a control background (UAS-Dcr2/+;GAL4 $4^{19-12}, U A S-C D 4-t d G F P /+$ ). The expression of two different RNAi constructs (v51227 or v108662) causes a decrease in the number of spiked protrusions. This decrease is enhanced in a genetic background heterozygous for a $f_{S}(1) h$ deletion $[D f(X) c 128]$, while one-copy of $D f(X) c 128$ alone did not cause a phenotype. $(B)$ Expression of $f_{s}(1) h$ RNAi also caused a defect in the behavioral response to a gentle touch stimulus. $(C, D)$ Extracellular electrophysiological recordings from class III neurons expressing $f_{S}(1) h$ RNAi indicated a reduction in the number of action potentials (APs) fired per second (no. of $\mathrm{AP} / \mathrm{s}$ ) for a mechanical stimulus of a particular intensity compared with baseline ( $\Delta$ no. of $\mathrm{AP} / \mathrm{s}$ ) (shown in $C$ ). $(D) \mathrm{A}$ representative recording trace is shown from control and RNAiexpressing cells for a stimulus of $20-\mu \mathrm{m}$ intensity. $(E, F)$ Images of single axon terminals from class III (ddaF) da neuron MARCM clones. Bar, $10 \mu \mathrm{m}$. Axon terminals from wild-type control $(E)$ or $f_{S}(1) h^{1112}$ mutant $(F)$ neurons appear similar. $(G)$ Quantification of axon terminal length shows no difference between control and $f_{S}(1) h^{1112}$ neurons. Values are reported as mean \pm SEM, with statistical significance reported as results of Student's $t$-test. 
2003; Li et al. 2004; Sugimura et al. 2004; Kim et al. 2006; Hattori et al. 2007; Crozatier and Vincent 2008). Moreover, the expression of $\mathrm{Ct}$ and Ss regulates class III da neuron spike morphology (Grueber et al. 2003; Kim et al. 2006). We observed a loss of Ct expression in $f_{S}(1) h^{1112}$ mutant class III da neuron clones (Fig. 5), which suggests that $f_{S}(1) h$ regulates the induction or maintenance of $\mathrm{Ct}$ expression throughout class III da neuron development. However, reintroducing Ct expression to class III da neuron $f_{S}(1) h^{1112}$ clones did not rescue the nearly absent spike morphology (Fig. 5). Therefore, the class III da neuron dendritic phenotype caused by loss of $f_{s}(1) h$ cannot be solely attributed to the loss of $\mathrm{Ct}$ protein. Since it is thought that $\mathrm{Ct}$ is a component of a genetic pathway responsible for subtype-specific dendritic arbor development, it is possible that $f_{S}(1) h$ regulates Ct expression as well as expression of genes necessary for the $\mathrm{Ct}$ pathway to affect dendritic morphology. Therefore, the relationship between $c t$ and $f_{s}(1) h$ does not appear to be a linear pathway, and $f_{S}(1) h$ might regulate both upstream and downstream components of $c t$. Our data indicating that $f_{S}(1) h$ is necessary for the Ct-induced overbranching and spike formation in class I da neuron dendrites (Fig. 6) support the idea that $f_{S}(1) h$ regulates the expression of downstream components of the $\mathrm{Ct}$ pathway, which are necessary for Ct-induced overbranching and spike formation. This hypothesis also explains why reintroducing $\mathrm{Ct}$ expression to $f_{S}(1) h^{1112}$ clones fails to rescue the dendrite phenotype. It is also known that $\mathrm{Ct}$ and Racl act synergistically to produce spike morphology (JinushiNakao et al. 2007). We examined Racl overexpression in a $f_{S}(1) h^{1112}$ mutant background and found that Racl expression significantly rescued the loss of spikes in class III da neurons (Fig. 5). However, Racl-induced overbranching in class I da neurons was not affected by $f_{S}(1) h^{1112}$ (Fig. 6). Therefore, $f_{S}(1) h$ does not appear to regulate genes downstream from $R a c 1$ but does regulate genes downstream from $c t$. Since these pathways are known to converge in order to regulate dendritic spike formation, our data suggest that $f_{S}(1) h$ may be a crucial link between these two pathways. One possible scenario is that $c t$ and Rac1 regulate parallel pathways, but $c t$ may regulate the level of Rac1 expression such that increased Rac1 expression facilitates the formation of spikes. In this model, our results support the hypothesis that $f_{S}(1) h$ is necessary for the $c t$ potentiation of Rac1 expression, which explains why increased expression of Rac1 with UAS-Rac1 causes a rescue of the class III da neuron dendritic phenotype in $f_{S}(1) h^{1112}$ mutants. Recent evidence indicates a role for reduced Rac1 expression in social defeat and depressive behavior in mice, possibly through regulating dendritic spine morphology (Golden et al. 2013). In these behavioral paradigms, reduced Rac1 expression occurred with altered epigenetic marks such that transcriptionally permissive histone $\mathrm{H} 3$ acetylation was reduced, while repressive histone $\mathrm{H} 3$ methylation was increased. Moreover, administering a class 1 HDAC inhibitor mitigated the reduced Rac1 expression. Thus, these data suggest that Rac1 expression can be regulated by histone acetylation. It is possible that epigenetic reader proteins, such as BET family proteins like
$f_{S}(1) h$, bind acetylated histone marks in the Rac1 promoter to recruit transcriptional machinery and in turn enhance Rac1 expression.

In addition, overexpression of $U A S-F s h-S$ in class I da neurons did not cause an overbranching phenotype similar to UAS-Ct (Fig. 6). In fact, there was no alteration of class I morphology, suggesting that Fsh-S is not sufficient to induce necessary components of the $c t$ pathway to alter dendrite morphology. However, overexpression of Fsh-S in class III (Fig. 1) and class IV (Supplemental Fig. S2) da neurons did cause a decrease in dendritic spike numbers. These data indicate that dendrite morphology may be sensitive to the amount of Fsh-S expression, which we confirmed by modulating the amount of overexpression by reducing GAL4/UAS activity with lower temperature (Supplemental Fig. S4). This may explain why we can achieve only a partial rescue of the $f_{S}(1) h^{1112}$ dendritic phenotype with UAS-Fsh-S expression and why overexpression causes a dendritic phenotype similar to the phenotype caused by loss of Fsh-S. In support of this expression level hypothesis, we observed that Fsh-S overexpression can reduce Ct-induced branching in class I da neurons (Supplemental Fig. S9). Since BRD2 is known to be part of a protein complex (Denis et al. 2006), it is possible overexpression causes a gain-of-function or dominant-negative effect by altering the availability of complex components.

Another possible explanation for the partial rescue of Fsh-S expression concerns the developmental timing of expression. Since these experiments were completed using MARCM, GAL80 is expressed until mitotic recombination occurs to generate the mutant clones. It is likely that GAL80 protein may persist for some time after the clones are formed, and the presence of GAL80 would block GAL4/UAS activity. Therefore, our UAS-induced Fsh-S expression may occur at a delayed stage in embryonic development, which could produce a partial rescue. In actuality, a combination of both expression level and developmental timing probably explains the partial rescue of the $f_{S}(1) h^{1112}$ phenotype.

While we focused on the role of $f_{S}(1) h$ in regulating class III da neuron dendrite morphology, we did observe phenotypes in other classes of the da neurons as well as expression of Fsh-S in all da neuron classes. In $f_{S}(1) h^{1112}$ mutants, we observed a loss of Ct expression in all da neurons that normally express Ct (classes II, III, and IV) (Fig. 2; Supplemental Fig. S7), suggesting that $f_{s}(1) h$ regulates $\mathrm{Ct}$ expression broadly among different neural subtypes. We also observed a loss of the class I-specific transcription factor $\mathrm{Ab}$ (Supplemental Fig. S10) and the class IV-specific transcription factor Kn/Col (Supplemental Fig. S11). Thus, it appears that $f_{S}(1) h$ can regulate the expression of subtype-specific gene expression among various neuron subtypes. The loss of $\mathrm{Ct}$ or the loss of $\mathrm{Kn} / \mathrm{Col}$ could explain the reduction in class IV da neuron dendritic arbor complexity, and this further illustrates the pleiotropic nature of the $f_{s}(1) h^{1112}$ phenotype. The loss of $\mathrm{Ab}$ from class I da neurons should produce an increase in dendritic complexity (Li et al. 2004), but interestingly, this did not occur in $f_{S}(1) h^{1112}$ mutants. Thus, these results consistently suggest that $f_{S}(1) h$ is necessary for 
dendritic arbor complexity, probably by regulating the expression of many different genes. In this manner, $f_{S}(1) h$ could act as a necessary gate for the gene expression responsible for establishing dendritic complexity.

How can $f_{S}(1) h$ regulate gene expression? Histone modifications are a diverse set of post-translational modifications that produce a code (Strahl and Allis 2000) whereby epigenetic reader proteins bind these modified histone tails with specificity for particular modifications, such as methylation, acetylation, or phosphorylation (Borrelli et al. 2008). Previous structural studies have shown that the bromodomains of BET family proteins form a hydrophobic pocket enveloping acetylated histone tails (Umehara et al. 2010a,b). Moreover, histone acetylation is largely, but not exclusively, regarded as a mark for transcriptional activation (Maze et al. 2013). Therefore, $f_{S}(1) h$ may be required for transcriptional activation of gene expression, which has been shown in vitro with respect to Ubx (Chang et al. 2007). Our data suggest that $f_{s}(1) h$ is required for $c t$ expression and is in agreement with the hypothesis that $f_{s}(1) h$ is a transcriptional activator. It is possible that expression of other genes in the $c t$ pathway also depends on histone acetylation modifications for transcriptional activation, and this activation may require Fsh-S. This would explain our observed nonlinear genetic relationship between $c t$ and $f_{s}(1) h$. In addition, our results indicate a necessary, but not sufficient, role of $f_{s}(1) h$ in regulating gene expression. This may indicate that BET family proteins require histone acetylation marks to be established but that these scaffold reader proteins do not actively alter histone tail modifications.

Histone modifications, termed the histone code (Strahl and Allis 2000), vary among different cell types and constitute a genome-wide mechanism for coordinating gene expression programs. This is intriguing because $f_{S}(1) h$ contains bromodomains that require histone acetylation to be first established at specific genomic regions in order to influence transcription at these regions. Our results suggest BET family proteins as candidates for reading this histone code to allow the development of dendritic complexity. It is important to note that although we observed many proteins with altered expression in $f_{s}(1) h^{1112}$ mutant da neurons, some proteins were unaltered, such as the mechanosensitive ion channel NompC (Supplemental Fig. S12). Furthermore, even though the Ct-induced overbranching in class I da neurons was blocked by $f_{s}(1) h^{1112}$ (Fig. 6), the Ct-induced NompC expression was normal (Supplemental Fig. S13). These data indicate some specificity to the action of $f_{S}(1) h$ in regulating dendritic morphology but not ion channel specification. It is possible that epigenetic "reader" proteins, such as the BET proteins, coordinate the activity of many genetic pathways but with relevance to a specific outcome, such as regulating dendritic arbor morphology. In this model, the epigenetic "readers" provide coordination and specificity of genome-wide histone marks to regulate particular aspects of neural cell biology. Moreover, it is conceivable that the specific genes regulated by $f_{S}(1) h$ could vary among different cell types depending on the cell typespecific histone code. This is supported by the different effects of UAS-Fsh-S overexpression in class I versus class III and IV da neurons as well as the loss of expression of cell type-specific transcription factors $(\mathrm{Ab}$ and $\mathrm{Kn} / \mathrm{Col})$ in $f_{S}(1) h^{1112}$ mutants. Currently, there is no atlas of the histone code for individual neural subpopulations. However, as the technology for conducting these types of analyses improves for distinct cell populations, it is conceivable that future studies can provide an answer to how cell type-specific histone modifications affect neural subtype-specific dendritic arbor morphologies.

\section{Dendritic arbor morphology contributes to mechanosensory neuron function}

Finally, our results suggest that the specific morphological shape of the class III da neuron dendrites is important for their ability to appropriately respond to sensory stimuli. Our results indicate that pathways regulating dendrite morphology, such as the $c t$ pathway, are reduced in $f_{S}(1) h$ mutants, but other pathways involved in axon morphogenesis or cell type-specific physiology, such as NompC channel expression, remain active. Moreover, the number of spike protrusions correlates with the number of APs produced in response to a mechanosensitive stimulus (Fig. 7). This was also observed in another study (Tsubouchi et al. 2012) involving manipulation of the number of spiked protrusions through modulating Racl activity. In that study, the gentle touch response increases as spike numbers increase, causing elevated calcium activity detectable with GCaMP fluorescence imaging. Conversely, decreasing the spike numbers results in a decrease of the gentle touch response and calcium activity. One potential caveat to this study is that Rac1 can modulate many aspects of dendritic cell biology through modulating actin cytoskeletal dynamics (Hotulainen and Hoogenraad 2010), and therefore it is unclear whether manipulating Racl activity alters the electrophysiological properties or localization of ion channels such as NompC. Our finding of a correlation between dendritic spike number and gentle touch/electrophysiological responses in $f_{s}(1) h$ mutant neurons with normal appearance of NompC expression implicates dendritic morphology in regulating touch sensitivity.

Interestingly, NompC is expressed in $f_{S}(1) h^{1112}$ mutants, and its distribution throughout the dendritic arbor resembles that of wild-type neurons (Supplemental Fig. S12). While nompC mutants lack a mechanosensory response (Yan et al. 2013), neurons lacking $f_{S}(1) h$ still respond to mechanical stimuli, but the magnitude of the response (number of APs) is reduced for a given stimulus intensity. At the behavioral level, this manifests as a reduced response to gentle touch. Therefore, our data suggest that the unique dendritic spike morphology of class III dendrites contributes to their mechanical sensitivity.

While various proteins involved in epigenetic regulation of gene expression have been implicated in dendrite morphogenesis, our study provides evidence that "readers" of acetylated histone marks regulate dendrite morphology by demonstrating the involvement of BET family proteins in this process. Given the complexity of achieving a compre- 
hensive view of molecularly defined neural subtypes, we need to identify genome-wide mechanisms for molecular diversity that regulate dendritic morphology in order to further understand how morphological diversity is specified. Epigenetic regulators are an intriguing possibility in this endeavor, and future studies comparing gene expression profiles in mutants for regulators of histone modifications among neurons with varied morphologies may be one step forward in answering this fundamental question.

\section{Materials and methods}

Fly stocks

All experimental fly crosses were maintained in circadian incubators (Darwin Chambers Company) at $25^{\circ} \mathrm{C}$ (or $20^{\circ} \mathrm{C}$ for GAL4 attenuation). The $f_{S}(1) h^{1112}$ allele was generated through EMS mutagenesis and mapped as previously described (Zheng et al. 2008). We generated UAS-Fsh-S-eGFP and UAS-huBRD2eGFP transgenes for rescue experiments with either the sequence of Fsh-S (Drosophila Genomics Resource Center [DGRC] clone LD26482, primers [5'] CGTACGTATATATCGTTGCAAC TTTTTAAC and [3'] ACCTGCTTCACTGTCGCTCGAGTC AC) or huBRD2 (Open Biosystems clone ID 6181728, primers [5'] ATGCTGCAAAACGTGACTCCCCACAATAAGCTCC or [3'] GCCTGAGTCTGAATCACTGGTGTCTG) using primers with $5^{\prime}$-attB1 and $3^{\prime}$-attB2 flanking sequences added for use in the Gateway cloning system (Invitrogen). The appropriate sequence was first subcloned into the pDONR-221 entry vector using BP-Clonase II (Invitrogen) and subsequently transferred to the pTWG vector (DGRC clone 1076) using LR-Clonase II (Invitrogen). The pTWG vector was modified to include an attB site for $\phi$ C-31-mediated integration (Bateman and Lee 2006) into flies containing the PBac\{y+-attP-3B\}VK00037 docking site (Bloomington Stock Center, 9752). Transgenic flies were created using the commercial service provided by Rainbow Transgenic, Inc. We used UAS-Ct.L (Grueber et al. 2003) and UAS-Rac1 (Bloomington Stock Center, 6293) for GAL4/UAS expression of $\mathrm{Ct}$ or Rac1 proteins, respectively. For RNAi-mediated knockdown, stocks v51227 and v108662 from the Vienna Drosophila RNAi Center (VDRC) stock center were used. The RNAi transgenes were expressed in class III neurons using GAL4 ${ }^{19-12}$ (gift from H.H. Lee, identified from the GAL4-enhancer trap collection of Dr. Ulrike Heberlein) (Xiang et al. 2010) in combination with UAS-CD4-tdGFP (Han et al. 2011) to visualize dendritic arbors and UAS-Dcr2 (Bloomington Stock Center, 24650) to enhance RNAi knockdown efficiency (Dietzl et al. 2007). GAL4/UAS expression in all da neurons was achieved using the pan-da neuron driver $G A L 4^{109(2) 80}$ (Gao et al. 1999) and in class I da neurons was achieved using GAL4 $4^{2-21}$ (Grueber et al. 2003).

\section{MARCM}

To generate MARCM clones, mutant $y w, F R T-19 A, f_{S}(1) h^{1112}$ or control yw,FRT-19A virgin female flies were mated to $y w, F R T$ 19A, tub-GAL80;sop-FLP, GAL4 ${ }^{109(2) 80}$, UAS-mCD8-GFP male flies. The sop-FLP transgene was generated and generously provided by Dr. Tadashi Uemura. Wandering, third instar larvae were collected, and da neuron clones were visualized through live imaging as described previously (Grueber et al. 2002).

\section{Immunohistochemistry}

Immunohistochemical staining was performed as reported previously (Grueber et al. 2002), except the mounting medium used was VectaShield (Vector Laboratories). The primary antibodies used were chicken anti-GFP (1:100; Aves Labs), rabbit anti-Ct (1:1000; clp2 epitope) (Grueber et al. 2003), mouse anti-Ct (1:20; Developmental Studies Hybridoma Bank [DSHB], F2 epitope) (Blochlinger et al. 1990), mouse anti-Abrupt (1:5; DSHB), mouse anti-kn/col (1:100; gift from A. Vincent, Center de Biologie du Développement, France) (Dubois et al. 2007), mouse antiNompC-NT (1:100; N-terminal epitope, a gift from J. Howard, Max Planck Institute, Dresden, Germany), rabbit anti-Fsh-S+L and anti-Fsh-L (1:100) (Chang et al. 2007), goat anti-HRP-Cy5 (1:100; Jackson ImmunoResearch), and rabbit anti-Nomp-EC (1:1000; generated against an extracellular epitope, amino acid residues 1469-1488; YenZym Antibodies, LLC). For antiNompC-EC staining, the tissue was nonpermeabilized with the exclusion of detergent throughout the immunostaining procedure. Secondary antibodies consisted of appropriate fluorescenceconjugated anti-donkey IgG (1:200; Jackson ImmunoResearch). Slides were imaged on a Leica SP5 confocal microscope using an oil immersion $40 \times$ objective.

\section{Dendrite/axon imaging}

For live imaging dendrite morphology, larvae were wholemounted on glass slides, sandwiched between the slide and coverglass in halocarbon oil to flatten and immobilize the larvae alive and intact (Grueber et al. 2002). A Leica SP5 confocal microscope equipped with a $20 \times$ oil immersion objective and an argon 488-nm emission laser was used to excite and image membrane-tagged fluorescent GFP expressed in da sensory neurons. Z-stacks containing the dendritic arbors of da sensory neurons were collected for analysis.

Single axon terminals were imaged as described previously (Kim et al. 2013) but with the following modifications: Larvae were filleted with the CNS intact and fixed in 4\% PFA for $30 \mathrm{~min}$ at room temperature. Larval filets were then mounted, and single da neuron axon terminals were imaged without immunostaining.

\section{Time-course/time-lapse imaging}

For time-sensitive experiments, staged embryo collections were performed on grape agar plates to synchronize groups of larvae (0 h AEL). For time-course experiments, larvae were grown on grape agar plates for 48,72 , or $96 \mathrm{~h} \mathrm{AEL}$ at $25^{\circ} \mathrm{C}$. da sensory neuron MARCM clones were imaged with a $20 \times$ oil immersion objective and at these various developmental stages. For timelapse imaging, synchronized larvae were collected at $72 \mathrm{~h} \mathrm{AEL}$ and imaged using a $40 \times$ oil immersion objective. Z-stacks were collected every $5 \mathrm{~min}$ over a 30 -min duration to visualize dendrite branch dynamics.

\section{Morphological analysis/quantification}

Morphological analysis of dendritic arbors was performed on maximum Z-projections of collected Z-stack image files. The arbors were reconstructed using the Simple Neurite Tracer plugin (Longair et al. 2011) of ImageJ FiJI (http://fiji.sc). Total dendrite length refers to the summed length of all dendrite branches from a single neuron reconstruction. Branch points were determined using the Analyze Skeleton function. Sholl analysis was performed using the Sholl Analysis feature of the Simple Neurite Tracer plugin. The class III spiked protrusions were manually counted using the Cell Counter ImageJ plugin (http://rsbweb.nih.gov/ij/plugins/cell-counter.html). For timelapse imaging, all terminal branch points within the field of view of projected Z-stacks were compared between consecutive 
time points and scored as growing (extending length), retracting (shortening length), or stable (no change in length). These events were counted across all time points over a 30 -min period and summed to determine the number of "total events." Growth, retraction, and stable branches were reported as a percentage of the "total events" sum.

Axon terminal length was quantified as described in Kim et al. (2013) using the Simple Neurite Tracer plugin of ImageJ FiJI.

\section{Behavioral assay/electrophysiology}

All behavioral and electrophysiological experiments were performed as reported previously (Yan et al. 2013). Briefly, for the gentle touch behavioral response assay, larvae were touched with an eyelash, and their subsequent behavior was categorically scored. The summed scores among larvae were averaged and reported as mean \pm SEM. Extracellular electrophysiological recordings were performed on class III da neurons visualized by $G A L 4^{19-12}, U A S-C D 4-t d G F P$ expression while delivering a mechanical stimulus by depressing the body wall cuticle a defined distance increment (intensity) with a sealed glass tip pipet. During the 1-sec duration of stimulus application, the number of APs was recorded and reported as the change in AP frequency ( $\Delta$ number of APs) compared with the spontaneous firing rate $1 \mathrm{sec}$ prior to stimulus presentation.

\section{Statistical analysis}

Statistical tests for significance were conducted using the SPSS program. When comparing two groups, a Student's $t$-test was used, whereas when comparing more than two groups, a one-way ANOVA followed by a post-hoc Tukey's test was performed.

\section{Acknowledgments}

We thank D.H. Huang for providing the anti-Fsh antibodies, and J. Howard for providing the anti-NompC-NT antibody. We thank L. Cheng for generating the anti-NompC-EC antibody. We thank A. Vincent for providing the anti-kn/col antibody. We thank S. Younger, S. Barbel, and T. Cheng for technical support. We thank W.J. Kim, D. Gorczyca, and all members of the Jan laboratory for discussion. We thank T. Uemura for fly lines. J.A.B. is the recipient of a National Science Foundation Graduate Research Fellowship Program under grant number 1144247. Z.Y. was the recipient of the Long-Term Fellowship from the Human Frontier Science Program. This work was supported by National Institutes of Health grant number R37NS040929 to Y.N.J. Y.N.J. and L.Y.J. are investigators of the Howard Hughes Medical Institute.

\section{References}

Andersen R, Li Y, Resseguie M, Brenman JE. 2005. Calcium/ calmodulin-dependent protein kinase II alters structural plasticity and cytoskeletal dynamics in Drosophila. J Neurosci 25: 8878-8888.

Bateman JR, Lee AM. 2006. Site-specific transformation of Drosophila via $\phi \mathrm{C} 31$ integrase-mediated cassette exchange. Genetics 173: 769-777.

Blochlinger K, Bodmer R, Jan LY, Jan YN. 1990. Patterns of expression of cut, a protein required for external sensory organ development in wild-type and cut mutant Drosophila embryos. Genes Dev 4: 1322-1331.

Borrelli E, Nestler EJ, Allis CD, Sassone-Corsi P. 2008. Decoding the epigenetic language of neuronal plasticity. Neuron 60: 961-974.
Chang Y-L, King B, Lin S-C, Kennison JA, Huang D-H. 2007. A double-bromodomain protein, FSH-S, activates the homeotic gene ultrabithorax through a critical promoter-proximal region. Mol Cell Biol 27: 5486-5498.

Corty MM, Matthews BJ, Grueber WB. 2009. Molecules and mechanisms of dendrite development in Drosophila. Development 136: 1049-1061.

Crozatier M, Vincent A. 2008. Control of multidendritic neuron differentiation in Drosophila: the role of Collier. Dev Biol 315: 232-242.

Denis GV, McComb ME, Faller DV, Sinha A, Romesser PB, Costello CE. 2006. Identification of transcription complexes that contain the double bromodomain protein $\mathrm{Brd} 2$ and chromatin remodeling machines. J Proteome Res 5: 502-511.

Dietzl G, Chen D, Schnorrer F, Su K-C, Barinova Y, Fellner M, Gasser B, Kinsey K, Oppel S, Scheiblauer S, et al. 2007. A genome-wide transgenic RNAi library for conditional gene inactivation in Drosophila. Nature 448: 151-156.

Dubois L, Enriquez J, Daburon V, Crozet F, Lebreton G, Crozatier M, Vincent A. 2007. Collier transcription in a single Drosophila muscle lineage: the combinatorial control of muscle identity. Development 134: 4347-4355.

Duffy JB. 2002. GAL4 system in Drosophila: a fly geneticist's Swiss army knife. Genesis 34: 1-15.

Gao FB, Brenman JE, Jan LY, Jan YN. 1999. Genes regulating dendritic outgrowth, branching, and routing in Drosophila. Genes Dev 13: 2549-2561.

Golden SA, Christoffel DI, Heshmati M, Hodes GE, Magida J, Davis K, Cahill ME, Dias C, Ribeiro E, Ables JL, et al. 2013. Epigenetic regulation of $\mathrm{RACl}$ induces synaptic remodeling in stress disorders and depression. Nat Med 19: 337-344.

Grueber WB, Jan LY, Jan YN. 2002. Tiling of the Drosophila epidermis by multidendritic sensory neurons. Development 129: $2867-2878$.

Grueber WB, Jan LY, Jan YN. 2003. Different levels of the homeodomain protein cut regulate distinct dendrite branching patterns of Drosophila multidendritic neurons. Cell 112: 805-818.

Guan J-S, Haggarty SJ, Giacometti E, Dannenberg J-H, Joseph N, Gao J, Nieland TJF, Zhou Y, Wang X, Mazitschek R, et al. 2009. HDAC2 negatively regulates memory formation and synaptic plasticity. Nature 459: 55-60.

Gyuris A, Donovan DJ, Seymour KA, Lovasco LA, Smilowitz NR, Halperin ALP, Klysik JE, Freiman RN. 2009. The chromatintargeting protein $\mathrm{Brd} 2$ is required for neural tube closure and embryogenesis. Biochim Biophys Acta 1789: 413-421.

Han C, Jan LY, Jan Y-N. 2011. Enhancer-driven membrane markers for analysis of nonautonomous mechanisms reveal neuron-glia interactions in Drosophila. Proc Natl Acad Sci 108: 9673-9678.

Hattori Y, Sugimura K, Uemura T. 2007. Selective expression of Knot/Collier, a transcriptional regulator of the EBF/Olf-1 family, endows the Drosophila sensory system with neuronal class-specific elaborated dendritic patterns. Genes Cells 12: 1011-1022.

Hattori Y, Usui T, Satoh D, Moriyama S, Shimono K, Itoh T, Shirahige K, Uemura T. 2013. Sensory-neuron subtype-specific transcriptional programs controlling dendrite morphogenesis: genome-wide analysis of Abrupt and Knot/Collier. Dev Cell 27: 530-544.

Häusser M, Mel B. 2003. Dendrites: bug or feature? Curr Opin Neurobiol 13: 372-383.

Ho L, Crabtree GR. 2010. Chromatin remodelling during development. Nature 463: 474-484.

Hobert O, Carrera I, Stefanakis N. 2010. The molecular and gene regulatory signature of a neuron. Trends Neurosci 33: 435-445. 
Hotulainen P, Hoogenraad CC. 2010. Actin in dendritic spines: connecting dynamics to function. J Cell Biol 189: 619-629.

Iyer EPR, Iyer SC, Sullivan L, Wang D, Meduri R, Graybeal LL, Cox DN. 2013. Functional genomic analyses of two morphologically distinct classes of Drosophila sensory neurons: post-mitotic roles of transcription factors in dendritic patterning. PLOS ONE 8: e72434.

Jan Y-N, Jan LY. 2010. Branching out: mechanisms of dendritic arborization. Nat Rev Neurosci 11: 316-328.

Jinushi-Nakao S, Arvind R, Amikura R, Kinameri E, Liu AW, Moore AW. 2007. Knot/Collier and cut control different aspects of dendrite cytoskeleton and synergize to define final arbor shape. Neuron 56: 963-978.

Kanno T, Kanno Y, Siegel RM, Jang MK, Lenardo MJ, Ozato K. 2004. Selective recognition of acetylated histones by bromodomain proteins visualized in living cells. Mol Cell 13: 33-43.

Kernan M, Cowan D, Zuker C. 1994. Genetic dissection of mechanosensory transduction: mechanoreception-defective mutations of Drosophila. Neuron 12: 1195-1206.

Kim MD, Jan LY, Jan YN. 2006. The bHLH-PAS protein Spineless is necessary for the diversification of dendrite morphology of Drosophila dendritic arborization neurons. Genes Dev 20: 2806-2819.

Kim JH, Wang X, Coolon R, Ye B. 2013. Dscam expression levels determine presynaptic arbor sizes in Drosophila sensory neurons. Neuron 78: 827-838.

Kirilly D, Wong JJL, Lim EKH, Wang Y, Zhang H, Wang C, Liao $\mathrm{Q}$, Wang $\mathrm{H}$, Liou $\mathrm{Y}-\mathrm{C}$, Wang $\mathrm{H}$, et al. 2011. Intrinsic epigenetic factors cooperate with the steroid hormone ecdysone to govern dendrite pruning in Drosophila. Neuron 72: 86-100.

Lee T, Luo L. 1999. Mosaic analysis with a repressible cell marker for studies of gene function in neuronal morphogenesis. Neuron 22: 451-461.

Li W, Wang F, Menut L, Gao F. 2004. BTB/POZ-zinc finger protein abrupt suppresses dendritic branching in a neuronal subtype-specific and dosage-dependent manner. Neuron 43: 823-834.

Longair MH, Baker DA, Armstrong JD. 2011. Simple Neurite Tracer: open source software for reconstruction, visualization and analysis of neuronal processes. Bioinformatics 27: $2453-2454$.

Lorenz S, Taylor KP, Gehrmann A, Becker T, Muhle H, Gresch M, Tauer U, Sander T, Stephani U. 2006. Association of BRD2 polymorphisms with photoparoxysmal response. Neurosci Lett 400: 135-139.

Maze I, Noh K-M, Allis CD. 2013. Histone regulation in the CNS: basic principles of epigenetic plasticity. Neuropsychopharmacology 38: 3-22.

Pal DK, Evgrafov OV, Tabares P, Zhang F, Durner M, Greenberg DA. 2003. BRD2 (RING3) is a probable major susceptibility gene for common juvenile myoclonic epilepsy. Am I Hum Genet 73: 261-270.

Parrish JZ, Kim MD, Jan LY, Jan YN. 2006. Genome-wide analyses identify transcription factors required for proper morphogenesis of Drosophila sensory neuron dendrites. Genes Dev 20: 820-835.

Parrish JZ, Emoto K, Jan LY, Jan YN. 2007. Polycomb genes interact with the tumor suppressor genes hippo and warts in the maintenance of Drosophila sensory neuron dendrites. Genes Dev 21: 956-972.

Parrish JZ, Xu P, Kim CC, Jan LY, Jan YN. 2009. The microRNA bantam functions in epithelial cells to regulate scaling growth of dendrite arbors in Drosophila sensory neurons. Neuron 63: $788-802$.
Petrij F, Giles RH, Dauwerse HG, Saris JJ, Hennekam RC, Masuno M, Tommerup N, van Ommen GJ, Goodman RH, Peters DJ. 1995. Rubinstein-Taybi syndrome caused by mutations in the transcriptional co-activator CBP. Nature 376: 348-351.

Puram SV, Bonni A. 2013. Cell-intrinsic drivers of dendrite morphogenesis. Development 140: 4657-4671.

Qi C, Liu S, Qin R, Zhang Y, Wang G, Shang Y, Wang Y, Liang J. 2014. Coordinated regulation of dendrite arborization by epigenetic factors CDYL and EZH2. I Neurosci 34: 44944508.

Shang E, Wang X, Wen D, Greenberg DA, Wolgemuth DJ. 2009. Double bromodomain-containing gene $\mathrm{Brd} 2$ is essential for embryonic development in mouse. Dev Dyn 238: 908-917.

Sinha A, Faller DV, Denis GV. 2005. Bromodomain analysis of Brd2-dependent transcriptional activation of cyclin A. Biochem J 387: 257-269.

Strahl BD, Allis CD. 2000. The language of covalent histone modifications. Nature 403: 41-45.

Sugimura K, Satoh D, Estes P, Crews S, Uemura T. 2004. Development of morphological diversity of dendrites in Drosophila by the BTB-zinc finger protein abrupt. Neuron 43: 809-822.

Sugino K, Hempel CM, Miller MN, Hattox AM, Shapiro P, Wu C, Huang ZJ, Nelson SB. 2006. Molecular taxonomy of major neuronal classes in the adult mouse forebrain. Nat Neurosci 9: 99-107.

Tea JS, Luo L. 2011. The chromatin remodeling factor Bap55 functions through the TIP60 complex to regulate olfactory projection neuron dendrite targeting. Neural Dev 6: 5.

Tea JS, Chihara T, Luo L. 2010. Histone deacetylase Rpd3 regulates olfactory projection neuron dendrite targeting via the transcription factor Prospero. J Neurosci 30: 99399946.

Tsubouchi A, Caldwell JC, Tracey WD. 2012. Dendritic filopodia, Ripped Pocket, NOMPC, and NMDARs contribute to the sense of touch in Drosophila larvae. Curr Biol 22: 2124-2134.

Umehara T, Nakamura Y, Jang MK, Nakano K, Tanaka A, Ozato K, Padmanabhan B, Yokoyama S. 2010a. Structural basis for acetylated histone $\mathrm{H} 4$ recognition by the human BRD2 bromodomain. I Biol Chem 285: 7610-7618.

Umehara T, Nakamura Y, Wakamori M, Ozato K, Yokoyama S, Padmanabhan B. 2010b. Structural implications for K5/K12di-acetylated histone $\mathrm{H} 4$ recognition by the second bromodomain of BRD2. FEBS Lett 584: 3901-3908.

Velíšek L, Shang E, Velíšková J, Chachua T, Macchiarulo S, Maglakelidze G, Wolgemuth DJ, Greenberg DA. 2011. GABAergic neuron deficit as an idiopathic generalized epilepsy mechanism: the role of BRD2 haploinsufficiency in juvenile myoclonic epilepsy. PLOS ONE 6: e23656.

Whitford KL, Dijkhuizen P, Polleux F, Ghosh A. 2002. Molecular control of cortical dendrite development. Annu Rev Neurosci 25: 127-149.

Wu JI, Lessard J, Olave IA, Qiu Z, Ghosh A, Graef IA, Crabtree GR. 2007. Regulation of dendritic development by neuronspecific chromatin remodeling complexes. Neuron 56: 94-108.

Xiang Y, Yuan Q, Vogt N, Looger LL, Jan LY, Jan YN. 2010. Light-avoidance-mediating photoreceptors tile the Drosophila larval body wall. Nature 468: 921-926.

Yan Z, Zhang W, He Y, Gorczyca D, Xiang Y, Cheng LE, Meltzer S, Jan LY, Jan YN. 2013. Drosophila NOMPC is a mechanotransduction channel subunit for gentle-touch sensation. Nature 493: 221-225.

Zheng Y, Wildonger J, Ye B, Zhang Y, Kita A, Younger SH, Zimmerman S, Jan LY, Jan YN. 2008. Dynein is required for polarized dendritic transport and uniform microtubule orientation in axons. Nat Cell Biol 10: 1172-1180. 


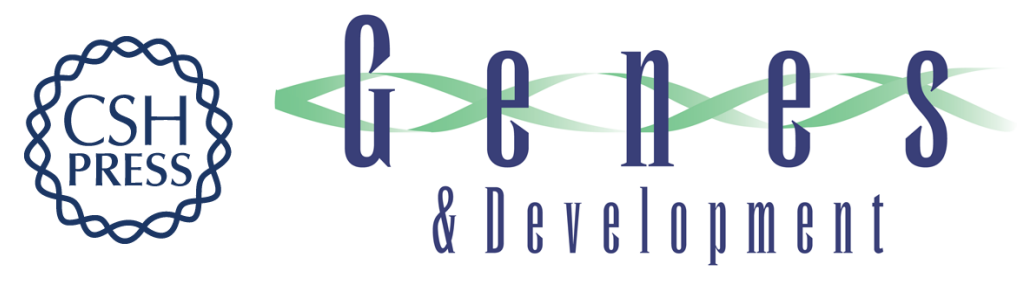

\section{Double-bromo and extraterminal (BET) domain proteins regulate dendrite morphology and mechanosensory function}

Joshua A. Bagley, Zhiqiang Yan, Wei Zhang, et al.

Genes Dev. 2014, 28:

Access the most recent version at doi:10.1101/gad.239962.114

Supplemental http://genesdev.cshlp.org/content/suppl/2014/09/02/28.17.1940.DC1
Material

References This article cites 60 articles, 16 of which can be accessed free at: http://genesdev.cshlp.org/content/28/17/1940.full.html\#ref-list-1

Creative This article is distributed exclusively by Cold Spring Harbor Laboratory Press for the first Commons License

Email Alerting Service six months after the full-issue publication date (see http://genesdev.cshlp.org/site/misc/terms.xhtml). After six months, it is available under a Creative Commons License (Attribution-NonCommercial 4.0 International), as described at http://creativecommons.org/licenses/by-nc/4.0/.

Receive free email alerts when new articles cite this article - sign up in the box at the top right corner of the article or click here.

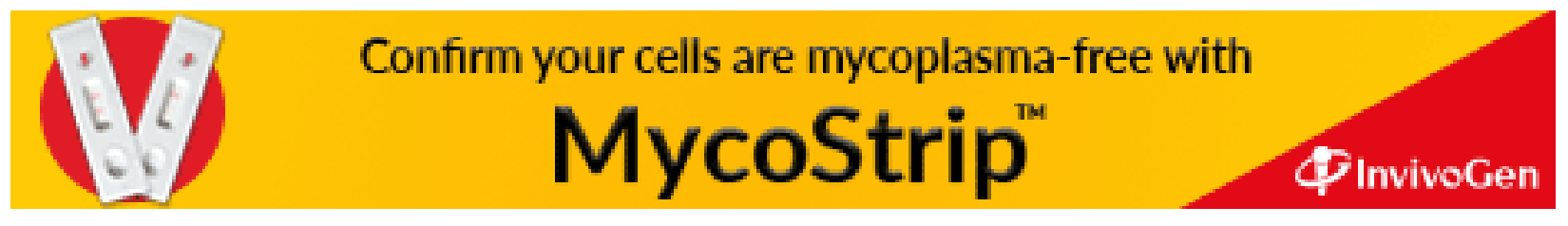

